\title{
Momentum and Energy Transport by Gravity Waves in Stochastically Driven Stratified Flows. Part I: Radiation of Gravity Waves from a Shear Layer
}

\author{
NikolaOs A. BAKAS \\ Harvard University, Cambridge, Massachusetts \\ Petros J. IoAnNou \\ National and Kapodistrian University of Athens, Athens, Greece
}

(Manuscript received 5 August 2005, in final form 24 August 2006)

\begin{abstract}
In this paper, the emission of internal gravity waves from a local westerly shear layer is studied. Thermal and/or vorticity forcing of the shear layer with a wide range of frequencies and scales can lead to strong emission of gravity waves in the region exterior to the shear layer. The shear flow not only passively filters and refracts the emitted wave spectrum, but also actively participates in the gravity wave emission in conjunction with the distributed forcing. This interaction leads to enhanced radiated momentum fluxes but more importantly to enhanced gravity wave energy fluxes. This enhanced emission power can be traced to the nonnormal growth of the perturbations in the shear region, that is, to the transfer of the kinetic energy of the mean shear flow to the emitted gravity waves. The emitted wave energy flux increases with shear and can become as large as 30 times greater than the corresponding flux emitted in the absence of a localized shear region.

Waves that have horizontal wavelengths larger than the depth of the shear layer radiate easterly momentum away, whereas the shorter waves are trapped in the shear region and deposit their momentum at their critical levels. The observed spectrum, as well as the physical mechanisms influencing the spectrum such as wave interference and Doppler shifting effects, is discussed. While for large Richardson numbers there is equipartition of momentum among a wide range of frequencies, most of the energy is found to be carried by waves having vertical wavelengths in a narrow band around the value of twice the depth of the region. It is shown that the waves that are emitted from the shear region have vertical wavelengths of the size of the shear region.
\end{abstract}

\section{Introduction}

Gravity waves play an important role in transporting energy and momentum and in influencing the general circulation and thermal structure of the atmosphere. Understanding the physical mechanisms that maintain the statistical equilibrium wave fluxes is a highly complex problem, as it involves knowledge of source characteristics, in addition to understanding and modeling of wave-wave and wave-mean flow interactions that influence and filter the emitted wave spectrum. The latter will be the focal point of this study.

Previous studies on wave-mean flow interactions have focused mainly on two effects of the background

Corresponding author address: Nikolaos Bakas, Geological Museum 403, 24 Oxford Street, Cambridge, MA 02138.

E-mail: bakas@fas.harvard.edu flow on waves. The first is dynamic excitation of gravity waves as a result of instability. A number of studies showed that the evolving vortex core of an unstable shear layer could couple strongly with radiating gravity waves in the case in which the region of strong shear is weakly stratified, whereas the buoyancy frequency is large in the far field (Sutherland and Peltier 1995; Sutherland 1996; Sutherland and Linden 1998), as well as in the case of Kelvin-Helmholtz instability (Fritts 1984; Chimonas and Grant 1984; Scinocca and Ford 2000).

The second, which is of primary interest here, is the effect of the mean flow on the spectrum of waves excited by exogenous sources. The atmospheric shear can alter the wave spectrum through critical level filtering and refraction (Beres et al. 2002, 2004; Fritts and Alexander 2003). It can also enhance wave generation in the presence of an external source such as convection (Clark et al. 1986; Lott 1997; Beres et al. 2004).

DOI: $10.1175 / \mathrm{JAS} 3905.1$ 
In particular Lott (1997) considered the evolution of small perturbations within a linearly stable stratified finite shear layer and found that transiently growing disturbances could lead to a significant flux of longwave propagating waves in the far field. These waves were found by Lott (1997) to have tapped energy from the mean shear. This result links the enhancement of waves to the nonnormality of the underlying dynamics that was also found to be important in breaking of inertia-gravity waves (Achatz and Schmitz 2006a,b) and many other geophysical examples (Farrell 1988, 1989; Farrell and Ioannou 1993a,b). An advantageous conceptual and mathematical framework for studying transiently growing nonmodal perturbations is the generalized stability theory (GST) developed by Farrell and Ioannou (1996). In the context of GST the various sources and nonlinearity provide through forcing the feedback perturbations required to maintain the variance while the energetic exchange between the forced background flow and the perturbations is included in the linearized dynamical operator.

Following this approach, we perform a GST analysis of a linear two-dimensional stably stratified model of an atmospheric shear flow that is externally forced. The purpose of the analysis is to investigate how a general shear flow influences and transforms an already existing spectrum of perturbations that is injected by the forcing inside the shear layer. We seek a parameterization for the forcing that will enable us to isolate the dynamics, while being minimally restrictive of the perturbation spectrum.

The physical processes and sources that give rise to this spectrum can be found in the detailed review paper of Fritts and Alexander (2003) and typically include topography, convection and adjustment of unbalanced flows. The set formed by all the sources spans a very wide range of phase speeds, wave frequencies, and vertical and horizontal scales. We therefore crudely parameterize the forcing as white noise in order to obtain the response of the system for all frequencies and scales. While such uncorrelated forcing is idealized, it lacks any bias and therefore allows the dynamical system to select the frequencies and structures that will play important role in momentum and energy transport.

Having specified the forcing, we assess how the waves are influenced by the mean flow by considering the simple model of a finite stably stratified shear region that has the advantage of admitting closed form solutions. This model also serves as an informative example of the prototype growth processes considered in this paper. In the companion paper (Bakas and Farrell 2006, manuscript submitted to J. Atmos. Sci., hereafter Part II) we apply the methods of GST to a linear two- dimensional jet, and examine the statistical equilibrium momentum and energy fluxes in and out of the jet, along with implications on the effect of the waves on the large-scale flow.

This paper is organized as follows. In section 2 we describe the linear evolution equations for perturbations in a stratified flow. Section 3 describes the temporal development of waves within a stably stratified infinite shear flow, focusing on the energetics and propagation properties involved. In section 4 we study a continuously forced finite shear layer bounded by two regions of uniform velocity, calculating initially the response to a monochromatic single point source, extending subsequently the analysis to a single point temporally uncorrelated source. In section 5 we investigate the response to vorticity and thermal stochastic forcing white both in space and time, covering also the case of a forcing that obeys a power-law behavior, assumed therefore red rather than white, and we end with a brief discussion and our conclusions in section 6 .

\section{Formulation}

Consider a flow of mean zonal velocity $U(z)$ varying only in the vertical in a hydrostatically balanced, stratified atmosphere of density $\rho=\rho_{m}+\rho(z)$, where $\rho_{m}$ is the mean density and $\rho(z)$ is the variation of the background density with height. Consider now velocity perturbations superposed on the mean flow in the zonal and vertical direction denoted $(u, w)$, respectively, and density and pressure perturbations superposed on the mean density and pressure fields denoted $(\rho, p)$, respectively. The linearized, nondimensional momentum and continuity equations that govern the evolution of the small perturbations are

$$
\begin{aligned}
\left(\partial_{t}+\frac{U(z)}{\sqrt{\mathrm{Ri}}} \partial_{x}\right) u+\frac{d U}{d z} \frac{w}{\sqrt{\mathrm{Ri}}}= & -\partial_{x} p-r(z) u \\
& +\frac{1}{\operatorname{Re}_{s}} \nabla^{2} u \\
\left(\partial_{t}+\frac{U(z)}{\sqrt{\mathrm{Ri}}} \partial_{x}\right) w= & -\partial_{z} p-\rho-r(z) w \\
& +\frac{1}{\operatorname{Re}_{s}} \nabla^{2} w \\
\left(\partial_{t}+\frac{U(z)}{\sqrt{\mathrm{Ri}}} \partial_{x}\right) \rho= & \frac{N^{2}}{N_{0}^{2}} w-r(z) \rho \\
& +\frac{1}{\operatorname{Re}_{s}} \nabla^{2} \rho, \\
\partial_{x} u+\partial_{z} w= & 0 .
\end{aligned}
$$


Time is nondimensionalized by a typical value of the Brunt-Väisälä frequency $N_{0}$ in the domain of the flow, the horizontal and vertical scales are nondimensionalized by a characteristic vertical scale $H$ and velocities are nondimensionalized by a typical value $V_{0}$. Pressure and density are nondimensionalized by $\rho_{m} H V_{0} N_{0}$ and $\rho_{m} V_{0} N_{0} / g$, respectively, where $g$ is the gravitational acceleration. The Richardson number and the Reynolds number are defined by $\mathrm{Ri}=N_{0}^{2} H^{2} / V_{0}^{2}$ and $\mathrm{Re}_{s}=$ $\rho_{m} H^{2} N_{0} / \mu$, where $\mu$ is the coefficient of viscosity. The Prandtl number has been chosen to be one; thus the coefficient of kinematic viscosity $\nu=\mu / \rho_{m}$ equals the coefficient of diffusion. The Brunt-Väisälä frequency will be herein considered constant and equal to its characteristic value $N^{2}=N_{0}^{2}$. The Rayleigh damping $r(z)$ and diffusion terms are only relevant to the numerical calculations presented in section 5 and further details will be provided therein. Eliminating pressure from (1) and (2) and taking advantage of the continuity equation to express the perturbation velocity field in terms of a streamfunction as $(u, w)=\left(\psi_{z}-\psi_{x}\right)$, we obtain the following evolution equations for the perturbation streamfunction, $\psi$, and density, $\rho$ :

$$
\begin{gathered}
\left(\partial_{t}+\frac{U(z)}{\sqrt{\mathrm{Ri}}} \partial_{x}+r(z)\right) \nabla^{2} \psi-\frac{1}{\sqrt{\mathrm{Ri}}} \partial_{x} \psi \frac{d^{2} U}{d z^{2}} \\
=-\frac{d r}{d z} \partial_{z} \psi+\partial_{x} \rho+\frac{1}{\mathrm{Re}_{s}} \nabla^{4} \psi, \\
\left(\partial_{t}+\frac{U(z)}{\sqrt{\mathrm{Ri}}} \partial_{x}+r(z)\right) \rho+\partial_{x} \psi=\frac{1}{\mathrm{Re}_{s}} \nabla^{2} \rho .
\end{gathered}
$$

\section{Gravity waves in stratified constant shear flow}

\section{a. Perturbation evolution of a single plane wave}

Consider an inviscid, stably stratified mean zonal constant shear flow $U(z)=z$. If we transform Eqs. (5)-(6) in the convected coordinate frame of reference that is moving with the background flow,

$$
\xi=x-\frac{z t}{\sqrt{\mathrm{Ri}}}, \quad \eta=z, \quad \tau=t,
$$

they become

$$
\begin{aligned}
\partial_{\tau}\left[\partial_{\xi}^{2}+\left(\partial_{\eta}-(\tau / \sqrt{\mathrm{Ri}}) \partial_{\xi}\right)^{2}\right] \psi & =\partial_{\xi} \rho, \\
\partial_{\tau} \rho & =-\partial_{\xi} \psi
\end{aligned}
$$

Equations (8)-(9) are now separable in $\xi$ and $\eta$ and a single Fourier component evolves as $\psi=\tilde{\psi}(\tau) e^{i k \xi} e^{i l \eta}$, where $k, l$ are the horizontal and vertical wavenum- bers in the convected coordinates, respectively. In the laboratory frame of reference the streamfunction is given by

$$
\psi=\tilde{\psi}(t) e^{i k x} e^{i(l-k t / \sqrt{\mathrm{Ri}}) z} .
$$

The time-varying amplitude of this Orr solution, $\tilde{\psi}(\tau)$, is determined from the evolution equation of the perturbation vorticity $\zeta$ :

$$
\frac{d^{2} \tilde{\zeta}}{d \tau^{2}}+\frac{1}{1+(l / k-\tau / \sqrt{\mathrm{Ri}})^{2}} \tilde{\zeta}=0,
$$

where

$$
\tilde{\psi}(\tau)=\frac{\tilde{\zeta}(\tau)}{k^{2}+(l-k \tau / \sqrt{\mathrm{Ri}})^{2}} .
$$

The solution of (11) can be analytically expressed using the standard Gauss hypergeometric functions (Hartman 1975) or can be accurately determined by numerical integration. The lines of constant phase of this wave rotate clockwise due to the linear decrease of the vertical wavenumber. If the plane wave initially leans against the shear, the Reynolds stress $\overline{u w}$, where the bar denotes an average over $x$ and $z$, is negative and remains negative up to the time $t_{v}=l \sqrt{\mathrm{Ri}} / k$ when the phase lines are vertical, for later times $t>t_{v}$ the plane wave tilts in the direction of the shear producing a positive Reynolds stress. Consequently, because the energy evolves according to

$$
\frac{d E}{d t}=-\frac{1}{\sqrt{\mathrm{Ri}}} \overline{u w},
$$

the perturbation energy density grows transiently for $t<t_{v}$, reaches a maximum at $t_{v}$ and decays for $t>t_{v}$

\section{b. Perturbation evolution of a wave group}

Consider now a wave packet of initial perturbations that consists of a spread $\Delta \mathbf{k}$ of wave vectors about a central vector $\mathbf{k}_{0}$ subject to $\|\Delta \mathbf{k}\| /\left\|\mathbf{k}_{0}\right\| \ll 1$. For simplicity we will restrict to a spread about $l_{0}$ only. The value of Richardson number plays a decisive role in the propagation of such wave packets (Hartman 1975).

For $\mathrm{Ri}<1 / 4$ the solutions ${ }^{1}$ to $(11)$ are purely monotonic, the wave packet will be subject only to minor distortion, and the perturbations described earlier grow transiently and finally surrender their energy to the

\footnotetext{
${ }^{1}$ This is a bulk Richardson number that for constant shear flow considered in this section coincides with the gradient Richardson number $\mathrm{Ri}_{g}$.
} 
mean flow in the neighborhood of their initial position. In the range $1 / 4 \leq \mathrm{Ri}$ Eq. (11) admits oscillating solutions, and for $\mathrm{Ri}<1$ wave packets move only a fraction of their central wavelength. For large Richardson numbers (i.e., Ri $\gg 1$ ), propagation clearly manifests, and a WKB approximation of (11) shows that the perturbations are internal gravity waves with a time-dependent vertical wavenumber $\tilde{l}=l-k t / \sqrt{\mathrm{Ri}}$ and time-dependent Doppler-shifted frequency, which satisfies the internal wave dispersion relation with the instantaneous value of the time dependent vertical wavenumber. For a wave packet, the WKB solution is reduced to (see appendix A for details)

$$
\begin{aligned}
\zeta(\tau)= & \frac{\tilde{A}}{\sqrt{\omega_{0}(\tau)}} \exp \left(i \int_{0}^{\tau} \omega_{0}(s) d s\right) \\
& +\frac{\tilde{B}}{\sqrt{\omega_{0}(\tau)}} \exp \left(-i \int_{0}^{\tau} \omega_{0}(s) d s\right),
\end{aligned}
$$

where

$$
\omega_{0}(\tau)=\frac{k_{0}}{\sqrt{k_{0}^{2}+\left(l_{0}-k_{0} \tau / \sqrt{\mathrm{Ri}}\right)^{2}}},
$$

and amplitudes $\tilde{A}, \tilde{B}$ depend on the initial conditions. The two terms in (14) represent two waves propagating in opposite directions. In appendix A, the group velocity of the waves is shown to be

$$
c_{g_{z}}= \pm \frac{k_{0}\left(l_{0}-k_{0} t / \sqrt{\mathrm{Ri}}\right)}{\left[k_{0}^{2}+\left(l_{0}-k_{0} t / \sqrt{\mathrm{Ri}}\right)^{2}\right]^{3 / 2}},
$$

where the plus sign is for the first term of (14) and the minus sign for the second term of (14).

Integrating Eq. (16) we obtain the vertical displacement of the center of the packet as a function of time:

$$
\begin{aligned}
\hat{z}= & \hat{z}(0) \pm \sqrt{\operatorname{Ri}}\left(\frac{1}{\sqrt{k_{0}^{2}+\left(l_{0}-k_{0} t / \sqrt{\mathrm{Ri}}\right)^{2}}}\right. \\
& \left.-\frac{1}{\sqrt{k_{0}^{2}+l_{0}^{2}}}\right) .
\end{aligned}
$$

From (16) and (17) we can see that under the assumption of a positive horizontal wavenumber $k_{0}$, an initial disturbance with positive central vertical wavenumber $l_{0}$ propagates upward (downward) for times $t<l_{0} \sqrt{\mathrm{Ri}} /$ $k_{0}$ reaching the maximum (minimum) height $\hat{z}_{\max }=$ $\pm \sqrt{\operatorname{Ri}}\left(1 / k_{0}-1 / \sqrt{k_{0}^{2}+l_{0}^{2}}\right)$, then the motion reverses and the packet asymptotically approaches a height $\hat{z}_{\infty}=$ $\pm \sqrt{\mathrm{Ri}} / \sqrt{k_{0}^{2}+l_{0}^{2}}$ lower/higher than its original position. It is worth noting that internal waves can be shown to propagate beyond their turning level due to nonlinear

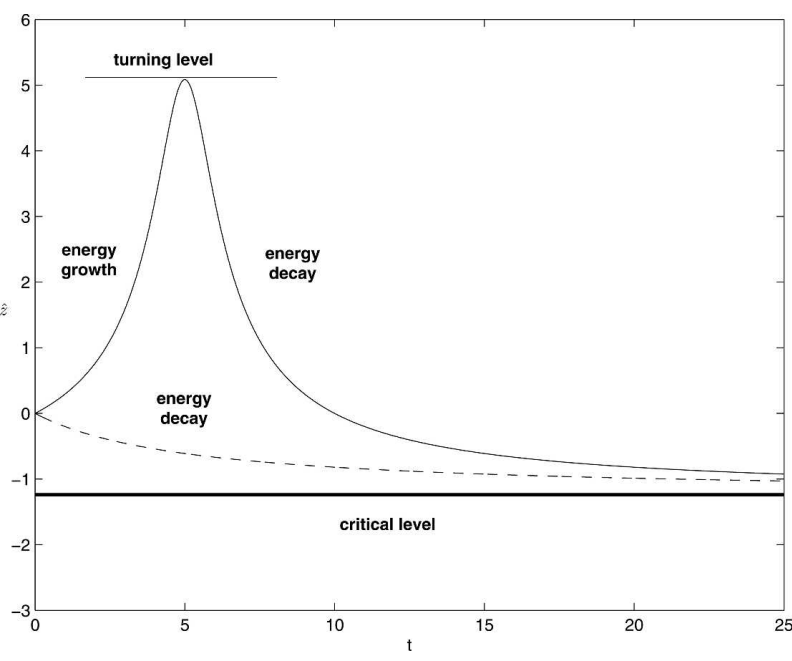

FIG. 1. The evolution of the center of a wavepacket $\hat{z}$ with time. The initial conditions are such that only the first term in Eq. (14) is nonzero, the Richardson number is chosen to be $\mathrm{Ri}=40$, and the central wavenumber is $\left(k_{0}, l_{0}\right)=(1,5)$ (solid line) and $\left(k_{0}, l_{0}\right)$ $=(1,-5)$ (dashed line). The thick solid line denotes the stagnation level for both cases and the phases of energy amplification and decay are also noted. When the initial conditions are such that the second term in (14) is nonzero, the path of the wave packet is the one shown here reflected about the $z=0$ axis.

interactions between the waves and the wave-induced flow (Sutherland 2000). Although the linearized analysis followed in this paper does not include such effects, the implications will be discussed in the next section. On the other hand, an initial disturbance with negative $l_{0}$, propagates downward/upward all the way down (up) to the minimum (maximum) height of $\hat{z}_{\max }=\mp \sqrt{\mathrm{Ri} /}$ $\sqrt{k_{0}^{2}+l_{0}^{2}}$. The trajectory of the center of the wave packet for both cases is shown in Fig. 1. Note that waves in weaker shear flows (lower Richardson numbers) or with larger initial tilts $l_{0} / k_{0}$ propagate to higher heights.

The evolution of the energy of the wave packet is given by

$$
\frac{\hat{E}(t)}{\hat{E}(0)}=\left(\frac{k_{0}^{2}+l_{0}^{2}}{k_{0}^{2}+\left(l_{0}-k_{0} t / \sqrt{\mathrm{Ri}}\right)^{2}}\right)^{1 / 2},
$$

which is the same expression found by Farrell and Ioannou (1993b) for a single Fourier coefficient. Even though wave action is conserved following the wave packet, for an initial positive vertical central wavenumber $l_{0}$ we have energy amplification during upward/ downward propagation and energy decay after reaching the turning level (where the group velocity changes sign). On the other hand, for a negative $l_{0}$ we have monotonic energy decay during the whole time of propagation. The phases of energy amplification and decay are also noted in Fig. 1. We finally note that the 
energy transfer between the wave packet and the mean flow occurs preferentially at the edges of the packet and not only from one point.

\section{Emission of gravity waves from a localized shear region}

While in constant shear flow perturbations may grow and decay in the manner described in the previous section; in flows with shear varying with height, the possibility arises that the energy tapped in regions of high shear may leak and lead to enhanced gravity wave activity in regions with predominantly high Richardson number. Lott (1997) considered the evolution of small perturbations within a stably stratified, finite shear layer and found that disturbances initially confined within the shear region grew transiently in the manner described in the previous section. He also found that, if the horizontal wavenumber of the initial perturbation was small, these disturbances led to spectacular gravity waves that propagated away from the shear layer, a fact that made their growth very persistent. This issue will also be addressed in the following sections. We will investigate the behavior of perturbations imposed in a stably stratified localized shear flow, with mean velocity given by

$$
U(z)= \begin{cases}V_{0}, & \text { for } z>z_{0} \\ V_{0} z / z_{0}, & \text { for }-z_{0}<z \leq z_{0}, \\ -V_{0}, & \text { for } z \leq-z_{0}\end{cases}
$$

where $z_{0}=1 / 2, V_{0}=1 / 2$ and constant Brunt-Väisälä frequency throughout the flow. This velocity profile is a shear layer localized in $-z_{0} \leq z \leq z_{0}$ and bounded by two regions of uniform velocity that extend from $-\infty \leq z \leq-z_{0}$ and $z_{0} \leq z \leq \infty$.

Before we treat the general stochastic excitation of gravity waves, it is both revealing and instructive to consider the excitation of gravity waves from a monochromatic single point thermal source. This case, which can be treated analytically (refer to appendix B), illuminates the salient processes of wave-mean flow interaction and filtering, which will prove to be of general validity. The details of the calculation can be found in appendix B. Here we will offer a heuristic and intuitive interpretation of the analytic results using the ideas which were introduced in the previous section.

For a monochromatic point thermal forcing of the form $f(x, z, t)=\delta(z) e^{i k(x-c t)}$, four possible evolution scenarios can be identified depending on the values of the phase speed $c$, Richardson number Ri, and wavenumber $k$. The wave packet trajectories that occur in each case are sketched in Figs. 2a,b and Figs. 3a,b.
The first case (Fig. 2a) occurs when the forced waves have both their turning level and critical level inside the shear region. For example, assume that $c<0$. Then the single point driving excites equally waves with phase lines initially leaning against $(l / k>0)$ and toward $(l / k<$ $0)$ the shear. The former propagate upward according to Fig. 1, their motion reverses at the turning level and asymptotically approach the stagnation level (critical level), which is lower than the point of excitation. It can be readily shown that the momentum flux contribution of such waves will be nonvanishing only below the forcing region. Moreover, the energy growth attained during the upward motion, according to (18), will be lost during the downward propagation toward the stagnation level. On the other hand, waves with phase lines leaning in the direction of the shear propagate downward toward their critical level surrendering their energy to the mean flow. The momentum flux contribution is again positive only below the forcing region, resulting in the total momentum flux distribution shown in Fig. 2c (dashed line).

The second case (Fig. 2b) occurs when the excited waves have their critical level but not their turning level inside the shear region. In this case the waves that lean against the shear can escape from the shear layer since they can reach the region of uniform velocity before their group velocity changes sign and in this way they can sustain the transient growth of energy that occurred during the upward/downward movement (depending on the sign of $c$ ). This can be illustrated by calculating the energy flux coming out of the shear [see Eq. (B10) of appendix B for more details] and comparing it with the corresponding flux in a similarly forced motionless atmosphere [this is also calculated in appendix B and given by Eq. (B13)]. The resulting ratio $\phi=\overline{p w} / \overline{p w}=$ $2.8>1$, which is noted in Fig. 2 d, quantifies the growth due to the presence of the shear layer. On the other hand, waves leaning toward the shear move to the critical level, and their energy monotonically decays, surrendering it to the mean flow as indicated by the positive total momentum flux distribution seen in Fig. 2 d.

The third case (Fig. 3a) occurs when the waves have a turning level but not a critical level inside the shear region. Westerly momentum is carried away from the shear as shown in Fig. 3c and is accompanied by a weak energy leakage $\left(\phi=\overline{p w} / \overline{p w} \bar{w}_{0}=0.38<1\right)$ attributed to the energy loss of the wave field in favor of the mean flow.

Nontrapping characterizes the fourth and final case as well (Fig. 3b) when the forced waves have neither a turning nor a critical level inside the shear region. Eastward and westward momentum is transported toward 
a

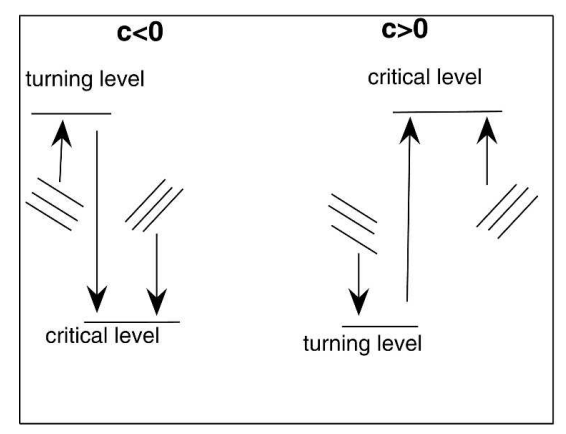

b

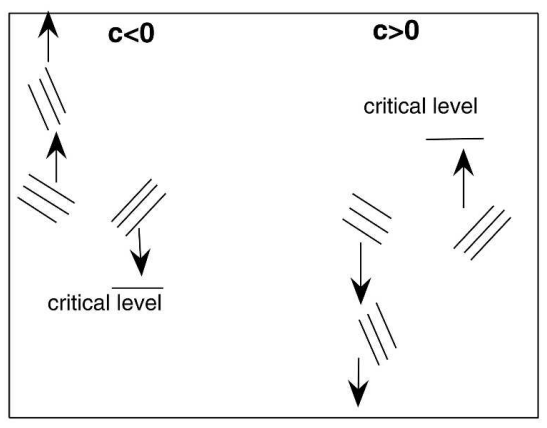

C

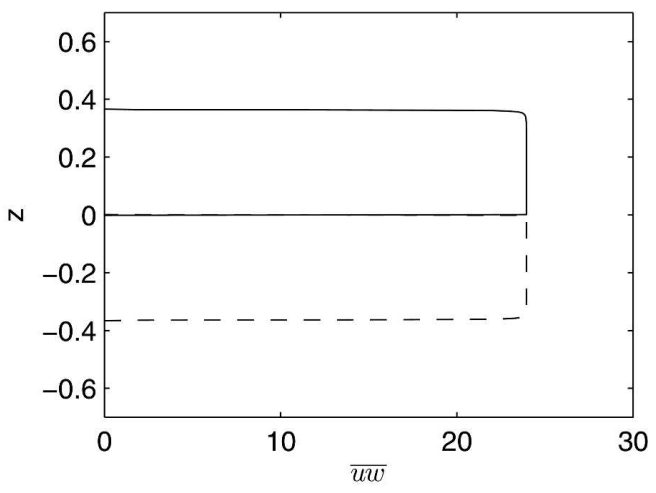

d

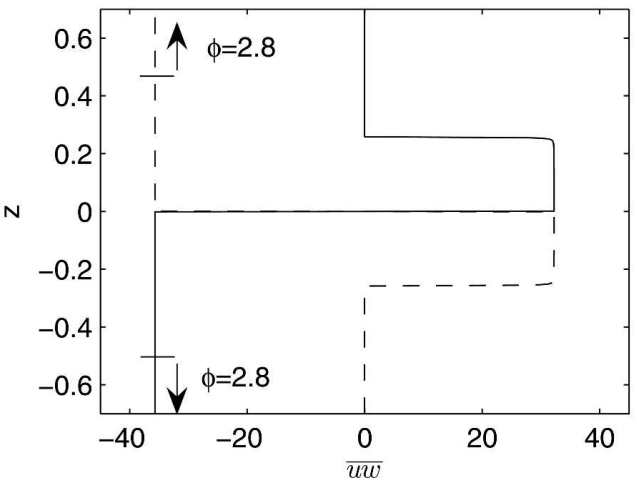

FIG. 2. Propagation diagrams of the excited waves in the shear region (19). (a) The waves have both a turning level and a critical level in the shear region and are consequently trapped. (b) The waves have a critical level in the shear layer but no turning level. In that case only the waves with phase lines tilted against the shear escape from the shear layer. (c) The resulting momentum flux distribution when both positively and negatively tilted waves of the type shown in (a) are excited. The cases shown are waves with phase velocity $c=0.149$ (solid line) and $c=-0.149$ (dashed line). (d) Momentum flux distribution when both positively and negatively tilted waves of the type shown in (b) are excited. The cases shown are waves with phase velocity $c=0.105$ (solid line) and $c=-0.105$ (dashed line). The Richardson number is $\mathrm{Ri}=6$ and the zonal wavenumber $k=3$. Noted in (d) is the outgoing energy flux, $\phi$, scaled by the outgoing flux in the absence of shear.

opposite directions, as seen in Fig. 3d, along with the enhanced or reduced energy fluxes noted in Fig. 3d.

It is worth noting that, depending on the values of Richardson number and horizontal wavenumber, at most three of the four possible cases can occur. When $\sqrt{\mathrm{Ri}} / k \leq 1 / 2$, regardless of the value of $c$, all waves are trapped and consequently only the first case occurs, as shown in the upper panel of Fig. 4. When $1 / 2<\sqrt{\mathrm{Ri} /}$ $k \leq 1$ the first three cases occur (middle panel), whereas when $1<\sqrt{\mathrm{Ri}} / k$ the last three cases (lower panel) occur.

Since for the last three cases all or part of the initial perturbation is emitted away from the shear region in the form of gravity waves, the question arises: Under broadband forcing at a point in the shear region, how much of the initial energy and momentum remains trapped in the region and what portion of it radiates to infinity?

To address this question, we force at the middle of the shear layer with a thermal forcing of the form

$$
f_{t}(x, z, t)=e^{i k x} \frac{1}{\alpha \sqrt{\pi}} e^{-z^{2} / \alpha^{2}} \eta(t),
$$

where $\eta(t)$ is a $\delta$ correlated white noise process. In the limit of $\alpha \rightarrow 0$ the forcing has a delta function vertical distribution. This forcing has, for nonzero $\alpha$, finite input variance and for the calculations that follow we choose $\alpha=0.025$. Since our dynamical system is asymptotically stable $(\mathrm{Ri}>1 / 4)$, all-second-order statistical quantities reach a steady value. The ensemble momentum and energy fluxes at this statistical equilibrium are given to a good approximation by 

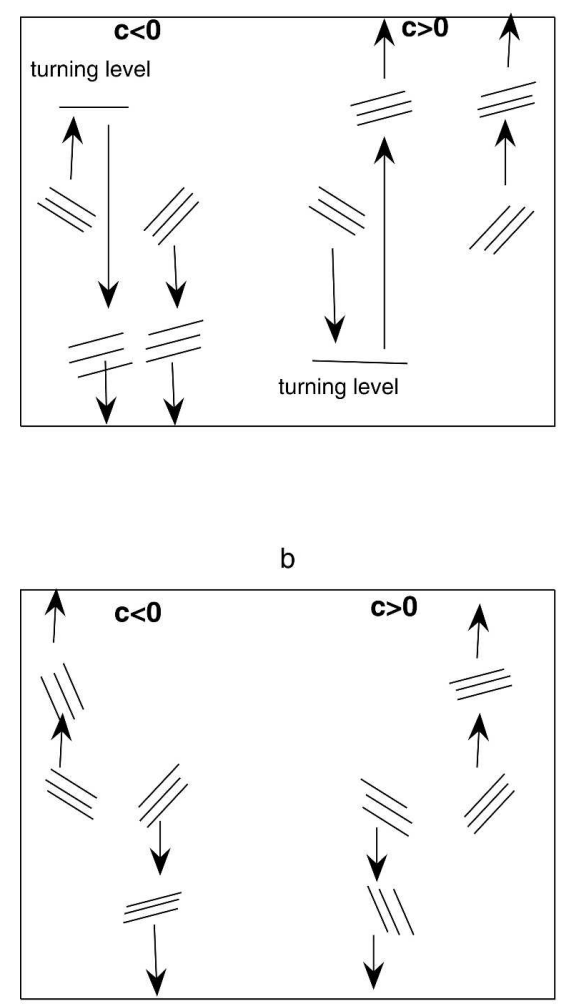

C

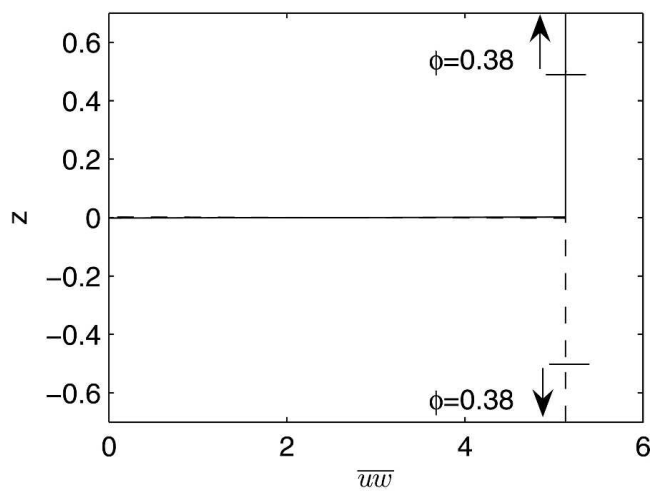

$d$

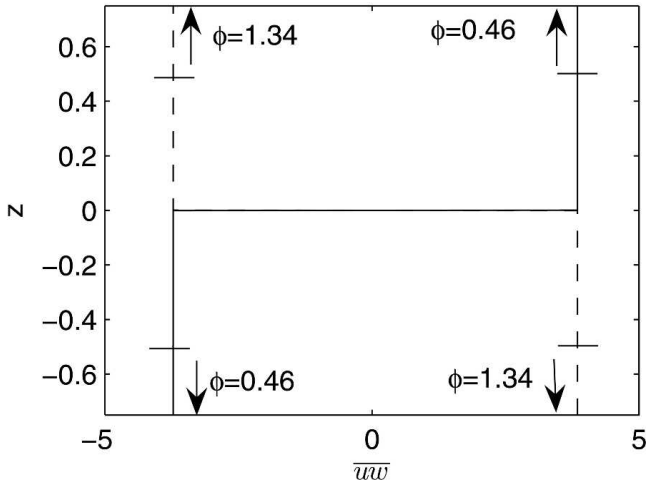

FIG. 3. Propagation diagrams of the excited waves in the shear region (19). (a) The waves have a turning level but not a critical level inside the shear region. (b) The excited waves have neither a critical nor a turning level inside the shear layer. (c) The resulting momentum flux distribution when both positively and negatively tilted waves of the type shown in (a) are excited. The cases shown are waves with phase velocity $c=0.277$ (solid line) and $c=-0.277$ (dashed line). The Richardson number is Ri $=6$ and the horizontal wavenumber $k=3$. (d) The momentum flux distribution when both positively and negatively tilted waves of the type shown in (b) are excited. The cases shown are waves with phase velocity $c=0.316$ (solid line) and $c=-0.316$ (dashed line). The Richardson number is $\mathrm{Ri}=10$ and the zonal wavenumber $k=1$. Noted in (c) and (d) are the outgoing energy fluxes, $\phi$, scaled by the outgoing fluxes in the absence of shear.

$$
[\langle\overline{u w}\rangle,\langle\overline{p w}\rangle]=\int_{-\infty}^{\infty}[\overline{u w}(\omega), \overline{p w}(\omega)] \hat{f f} * d \omega,
$$

where $\overline{u w}(\omega), \overline{p w}(\omega)$ are given by Eqs. (B9)-(B10) and

$$
\begin{aligned}
\hat{f} & =\frac{1}{\sqrt{2 \pi}} \int_{-\infty}^{\infty} \frac{1}{\alpha \sqrt{\pi}} e^{-z^{2} / \alpha^{2}} e^{-i l z} d z \\
& =\frac{1}{\sqrt{2 \pi}} e^{-\left(k^{2} \mathrm{Ri} / \omega^{2}-k^{2}\right) \alpha^{2} / 4} .
\end{aligned}
$$

The resulting $\langle\overline{u w}\rangle$ at statistical equilibrium for $\mathrm{Ri}=10$ and $k=1$ (which satisfies $1<\sqrt{\mathrm{Ri}} / k$ ) is plotted in the left panel of Fig. 5.

Although all frequencies are equally excited owing to the temporally uncorrelated forcing, the response (for a specific horizontal wavenumber), which is inversely proportional to $c$, favors low frequency waves that have a critical level but not a turning level inside the shear region, as illustrated in the middle and lower panels of Fig. 4. This result is verified by the large peak of $\overline{u w}$ and $\overline{p w}$ for low frequencies, which are plotted in the upper and lower right panels of Fig. 5. The excess energy gained by negative $c$ waves observed in the lower right panel of Fig. 5 compensates for the energy loss of positive $c$ waves leading to an overall energy flux increase of $\phi=3$. Despite the energy amplification occurring inside the shear zone, we cannot extract more eastward or westward momentum in the presence of shear [the curves with (solid line) and without shear (dashed line) almost coincide in the upper right panel of Fig. 5].

This result can be traced to two major factors: The first is that, above the forcing region, the momentum 

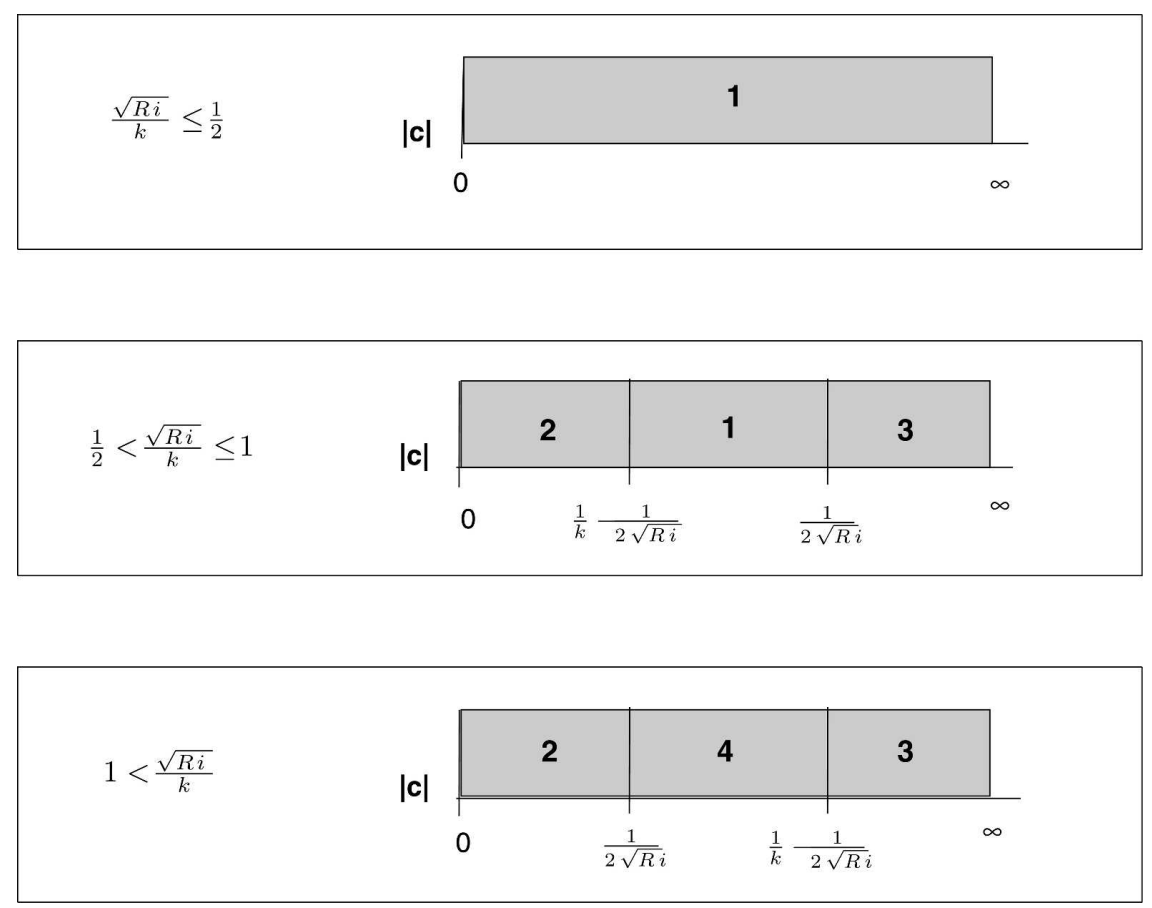

FIG. 4. Schematic representation of the various possible cases depending on the values of Ri, $k$, and $c$. For (upper) $\sqrt{\mathrm{Ri}} / k \leq 1 / 2$ all waves independent of the value of $c$ are trapped and only the first case (denoted by number 1 ) holds, for (middle) $1 / 2<\sqrt{\mathrm{Ri}} / k \leq 1$ the first three cases exist (denoted by $1,2,3$, respectively), and finally for (lower) $1<\sqrt{\mathrm{Ri}} / k$ the last three hold (denoted by 2,3, 4, respectively).

flux of waves that do not have a critical level inside the shear region is nondivergent according to the EliassenPalm (1961) theorem and independent of the mean flow. The second is that the forcing region occupies almost only a single point and the extracted momentum flux at the source region proved to have almost the same value regardless of the background flow. However, this is not the case for lower Richardson numbers, where extraction of more momentum even for such a localized source region is realized. The large net excess easterly momentum leakage observed in the left panel of Fig. 5 is associated with the critical level filtering of trapped waves, which is clearly demonstrated in the upper right panel of Fig. 5 by the absence of phase speeds in the range $0<c<0.16$ from the wave spectra. The filtering is accompanied by the resulting momentum deposition in the vicinity of $z=0$ that induces the sharp increase of $\langle\overline{u w}\rangle$ in the center of the shear layer. Sutherland (2000) studied the propagation of compact wave packets across a hyperbolic tangent shear layer and showed that due to nonlinear interactions between the waves and the mean flow, the wave packets could tunnel through their turning level and evade internal reflection. Our results are expected to be weakly influenced by such nonlinear effects due to the fact that waves having a turning level inside the shear region correspond to high frequencies where the response to the thermal forcing is small. We finally note that for $1 / 2$ $<\sqrt{\mathrm{Ri}} / k \leq 1$ we obtain similar results.

\section{Emission from spatially and temporally uncorrelated stochastic forcing}

\section{a. The case of temporally uncorrelated stochastic forcing}

We now consider stochastic excitations of the shear region with temporally and spatially broadband forcing that injects exogenous vorticity and thermal fluctuations with a wide range of frequencies and scales. The response to purely thermal and to spanwise vorticity forcing was calculated. Since there were minor differences when the forcings were normalized to have the same input variance, the results presented in this section correspond to a purely thermal excitation to facilitate comparison with results presented in the previous section.

It is advantageous for the analysis to express the perturbation equations (5) and (6) in the form

$$
\frac{d \mathbf{x}}{d t}=\mathbf{A x}+\mathbf{F f}(t),
$$

where $\mathbf{x}=[\psi(t), \rho(t)]^{\mathrm{T}}$ is the state vector, and 

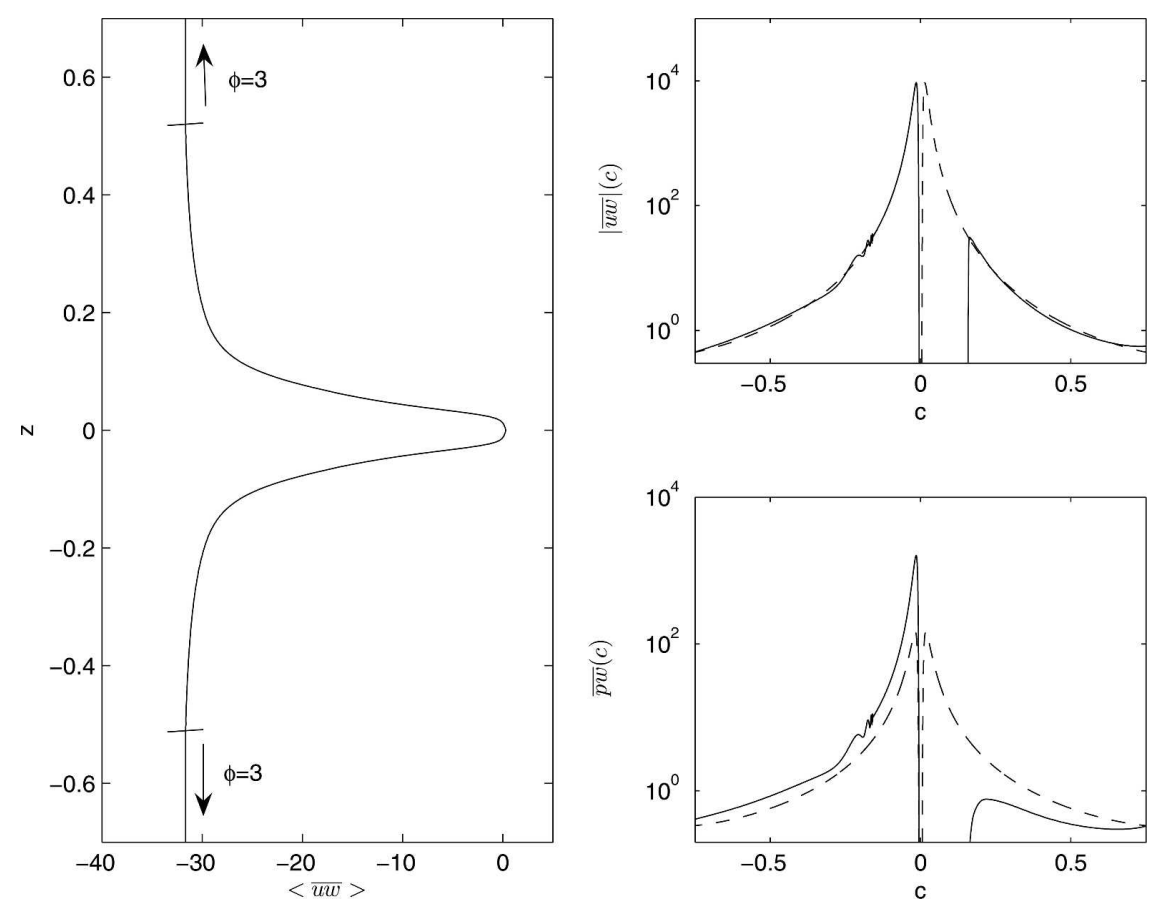

FIG. 5. (left) The ensemble momentum flux $\langle\overline{u w}\rangle$ variation with height for uncorrelated forcing in time. The Richardson number is $\mathrm{Ri}=10$ and the zonal wavenumber is $k=1(1<$ $\sqrt{\mathrm{Ri}} / k)$. The outgoing ensemble energy flux $\langle\overline{p w}\rangle$ calculated at $z= \pm 1$ and scaled by the corresponding flux $\left\langle\overline{p w}_{0}\right\rangle$ in the absence of the shear flow is noted as $\phi$. (right) The resulting distribution of the absolute value of momentum (upper right) and energy (lower right) fluxes at $z=1$ across wave phase speeds for $\mathrm{Ri}=10$ and $k=1$. The corresponding distribution of fluxes for a motionless atmosphere is also plotted (dashed lines) for reference. Note that the two lines coincide in the upper right panel for $c<0$ and for $c>0.16$ and that the corresponding distribution of fluxes at $\mathrm{z}=-1$ would be the symmetric counterpart of the one shown in the right panels with respect to the $c=0$ axis.

$$
\mathbf{A}=\left(\begin{array}{cc}
\nabla^{-2}\left(-\left(\frac{U}{\sqrt{\mathrm{Ri}}} \partial_{x}+r\right) \nabla^{2}+\frac{1}{\left.\sqrt{\mathrm{Ri}} \frac{d^{2} U}{d z^{2}} \partial_{x}-\frac{d r}{d z} \partial_{z}+\frac{1}{\mathrm{Re}_{s}} \nabla^{4}\right)}\right. & \nabla^{-2} \partial_{x} \\
-\partial_{x} & -\left(\frac{U}{\sqrt{\mathrm{Ri}}} \partial_{x}+r\right)+\frac{1}{\mathrm{Re}_{s}} \nabla^{2}
\end{array}\right),
$$

is the dynamical operator. By discretizing the differential operators we interpret the above equation as a matrix equation in which the state becomes a column vec- tor, the matrix $\mathbf{F}$ determines the spatial distribution of the forcing, and $\mathbf{f}(t)$ is a random vector function of time.

The mean velocity profile

$$
U(z)=\left\{\begin{array}{cl}
z, & 0 \leq z<z_{3} \\
\frac{z_{3}+z_{4}}{2}+\left(z_{3}-z_{4}\right) f\left(\frac{z-z_{4}}{z_{3}-z_{4}}\right), & z_{3} \leq z<z_{4} \\
\frac{z_{3}+z_{4}}{2}, & z \geq z_{4}
\end{array}\right.
$$




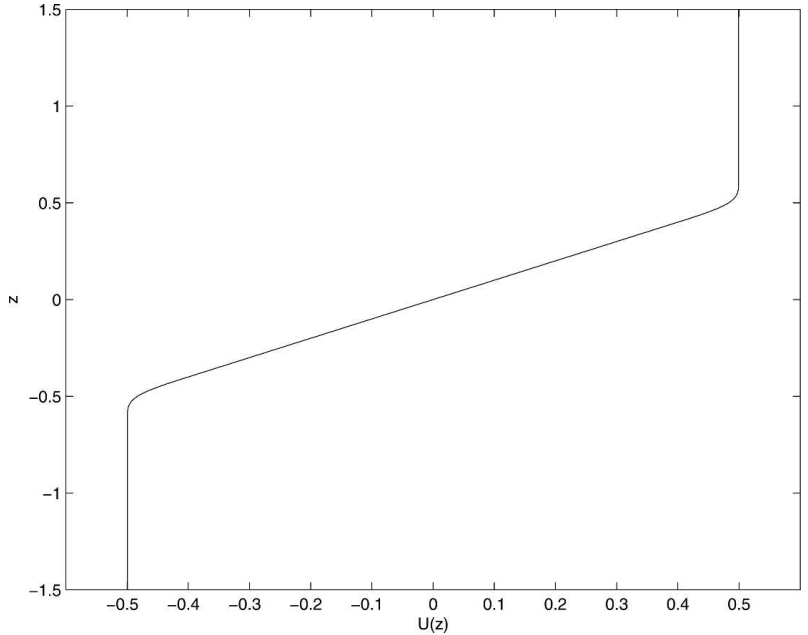

FIG. 6. Velocity vertical profile consisting of a shear region bounded by two domains of uniform velocity flow. For the profile shown $z_{3}=0.4, z_{4}=0.6$.

will be used with

$$
f(x)=\left\{\begin{array}{cc}
\frac{2}{3} x^{3}, & 0 \leq x<\frac{1}{2} \\
-\frac{2}{3} x^{3}+2 x^{2}-x+\frac{1}{6}, & \frac{1}{2} \leq x<1
\end{array} .\right.
$$

This profile, which is plotted in Fig. 6, was introduced by Lindzen and Barker (1985) and is antisymmetric with respect to the origin; that is, $U(-z)=-U(z)$. It differs from the profile given in (19) only in that the corners at the edges of the shear region have been smoothed out. We note that numerical tests done with a hyperbolic tangent profile showed that the results presented in this section are not sensitively dependent on the details of the velocity profile. The typical values of length and velocities used to nondimensionalize the equations are the width of the shear layer $H=2 z_{4}$ and the difference in velocities of the upper and lower regions $V_{0}=z_{3}+z_{4}$. We impose radiation conditions at infinity by inserting sponge layers at the top and the bottom through the introduction of the vertically varying Rayleigh damping:

$$
\begin{aligned}
r(z)= & a_{r}\left\{1+\tanh \left[\left(z-z_{\text {upper }}\right) / \delta_{r}\right]\right. \\
& \left.+\left(1-\tanh \left[\left(z-z_{\text {lower }}\right) / \delta_{r}\right]\right)\right\}
\end{aligned}
$$

in which $a_{r}$ is the amplitude of damping of the sponge layer, $z_{\text {upper }}$ and $z_{\text {lower }}$ are the vertical heights of the sponge, and $\delta_{r}$ determines the width of the transition into the sponge layer. We have selected sponge layer parameter values that resulted to no appreciable spurious back-reflection from the variation of $r(z)$.
We impose a spatially uncorrelated driving by choosing the columns of $\mathbf{F}$ to be $\hat{H}(z) \sin (2 n \pi z)$ and $\hat{H}(z)$ $\cos (2 n \pi z)$ for $n=1,2, \ldots$, where

$$
\hat{H}(z)= \begin{cases}1, & |z| \leq 1 / 2 \\ 0, & |z|>1 / 2\end{cases}
$$

is a "hat" function that restricts the forcing within the shear region. Since we do not want to force scales below the resolved ones, we truncate the forcing structures at the $n$ that corresponds to the largest resolved wavenumber, which for our grid point resolution is $n=10$. A small amount of diffusion is also introduced $\left(\operatorname{Re}_{s}=10^{5}\right)$ to serve as a sink for energy cascaded to unresolved scales.

The random vector function $\mathbf{f}$ is a $\delta$-correlated white noise process with zero ensemble mean and unit ensemble covariance and its components satisfy

$$
\left\langle f_{i}\left(t_{1}\right) f_{j}\left(t_{2}\right)\right\rangle=\delta_{i j} \delta\left(t_{1}-t_{2}\right)
$$

We also note that the forcing structures (given by the columns of $\mathbf{F}$ ) will be herein normalized so that $\int_{-1 / 2}^{1 / 2}$ $\mathbf{F}^{\dagger} \mathbf{M F} d z=1$, where $\mathbf{M}$ is the energy metric used to define the perturbation energy as the inner product: $E=\mathbf{x}^{\dagger} \mathbf{M} \mathbf{x}$ and $\mathbf{F}$ denotes from now on the normalized forcing structure matrix.

If we transform Eqs. (23) and (29) in the Fourier space of frequencies, the response of the system at frequency $\omega$ can be expressed as

$$
\hat{\mathbf{x}}(\omega)=\mathbf{R}(\omega) \mathbf{F} \hat{\mathbf{f}},
$$

where $\hat{\mathbf{x}}, \hat{\mathbf{f}}$ are respectively the Fourier transforms of $\mathbf{x}$ and $\mathbf{f}, \mathbf{R}(\omega)=(i \omega \mathbf{I}-\mathbf{A})^{-1}$ is the resolvent, and $\mathbf{I}$ is the identity matrix. Equation (29) equivalently becomes

$$
\left\langle\hat{f}_{i}\left(\omega_{1}\right) \hat{f}_{j}\left(\omega_{2}\right)\right\rangle=\delta_{i j} \delta\left(\omega_{1}-\omega_{2}\right),
$$

and the response covariance matrix at statistical equilibrium is

$$
\left\langle\mathbf{x x}^{\dagger}\right\rangle=\frac{1}{2 \pi} \int_{-\infty}^{\infty} \mathbf{F}(\omega) d \omega,
$$

where $\mathbf{F}(\omega)=\mathbf{R}(\omega) \mathbf{F} \mathbf{F}^{\dagger} \mathbf{R}^{\dagger}(\omega)$ (cf. Farrell and Ioannou 1996), and $\dagger$ denotes the Hermitian transpose. Pressure $p$ and horizontal and vertical velocities $u, w$ can be expressed in terms of $\mathbf{x}$ via linear operators $\mathbf{P}, \mathbf{U}$, and $\mathbf{W}$ (thus $\mathbf{P}=\mathbf{P} \mathbf{x}, \mathbf{u}=\mathbf{U} \mathbf{x}$, and $\mathbf{w}=\mathbf{W} \mathbf{x}$ ) yielding the following expressions for the momentum and energy fluxes at equilibrium:

$$
\left.\left[\overline{\left\langle\mathbf{u w}^{\dagger}\right\rangle}, \overline{\left\langle\mathbf{p w}^{\dagger}\right.}\right\rangle\right]=\left[\operatorname{diag}\left(\mathbf{U}\left\langle\mathbf{x} \mathbf{x}^{\dagger}\right\rangle \mathbf{W}^{\dagger}\right), \operatorname{diag}\left(\mathbf{P}\left\langle\mathbf{x} \mathbf{x}^{\dagger}\right\rangle \mathbf{W}^{\dagger}\right)\right],
$$



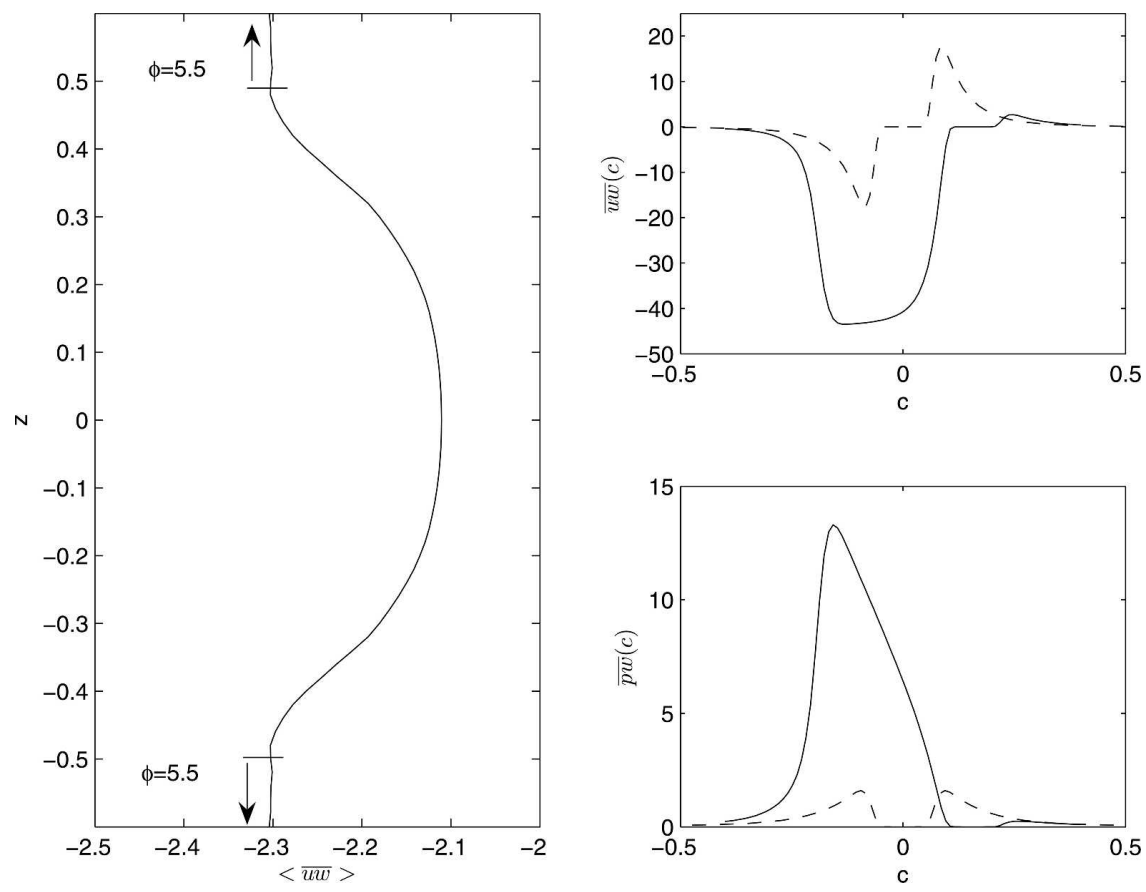

FIG. 7. (left) Integrated momentum flux $\langle\overline{u w}\rangle$ over all frequencies as a function of height for uncorrelated forcing in space and time. The Richardson number is $\mathrm{Ri}=10$ and the zonal wavenumber is $k=1$. The outgoing ensemble energy flux $\langle\overline{p w}\rangle$ calculated at $z= \pm 1$ (located outside the shear region) and scaled by the corresponding flux $\left\langle\overline{p w_{0}}\right\rangle$ in the absence of the shear flow is noted as $\phi$. (right) Distribution of $\overline{u w}$ (upper right) and $\langle\overline{p w}\rangle$ (lower right) at $z$ $=1$ with wave phase speeds. The corresponding distribution of fluxes for a motionless atmosphere is also plotted (dashed lines) for reference. It is isotropic with equal amounts of eastward and westward fluxes due to the eastward-westward symmetry.

where diag denotes the diagonal elements of a matrix. Equivalently, the equilibrium energy and momentum flux can be expressed in terms of the frequency response as

$$
\left[\left\langle\overline{\left.\mathbf{u w}^{\dagger}\right\rangle},\left\langle\overline{\left.\mathbf{p w}^{\dagger}\right\rangle}\right]=\frac{1}{2 \pi} \int_{-\infty}^{\infty}\left[\operatorname{diag}\left(\mathbf{U R}(\omega) \mathbf{F F}^{\dagger} \mathbf{R}^{\dagger}(\omega) \mathbf{W}^{\dagger}\right), \operatorname{diag}\left(\mathbf{P R}(\omega) \mathbf{F F}^{\dagger} \mathbf{R}^{\dagger}(\omega) \mathbf{W}^{\dagger}\right)\right] d \omega .\right.\right.
$$

The vertical distribution of the resulting momentum flux $\langle\overline{u w}\rangle$ at equilibrium is plotted in Fig. 7 (left panel). The distribution is very similar to the momentum flux distribution produced by a stochastic point source (cf. left panel of Fig. 5) indicating that the salient features of the dynamics have been captured by the simplified analysis. The quantitative differences in the response are related to two influencing factors: The first is the scaling of the forcing that imparts, in this case, on each Fourier component a unit of energy. The second is that the amplitude of the forced waves is a decreasing function of the width of the forcing region (Lindzen 1966) and consequently a reduced response is expected when the forcing region extends to the whole shear layer.
While the vertical distribution of the total momentum and energy flux are similar in the two cases, the phase speed spectra of $\overline{u w}$ and $\overline{p w}$ plotted in Fig. 7 (right panels) and Fig. 5 (right panels), are quite different. From Eq. (B3) the response is proportional to

$$
\frac{\mathrm{Ri}}{i k(U(z)-c \sqrt{\mathrm{Ri}})^{2}}=\frac{k}{i \hat{\omega}^{2}}=\frac{k^{2}+l^{2}}{i k},
$$

where $\hat{\omega}=k / \sqrt{k^{2}+l^{2}}$ is the intrinsic frequency. For a motionless atmosphere and in the inviscid limit this would lead to a sharp peak at the phase speed c corresponding to the largest forced vertical wavenumber 
$l_{0}=20 \pi ;^{2}$ that is, a sharp peak at phase speed $c=$ $\pm 1 / \sqrt{k^{2}+l_{0}^{2}}= \pm 0.016$. Such short wavelength waves have small group velocities and are attenuated by the small amount of diffusion in the model that shifts the peak to $c= \pm 0.08$ (dashed line). But in the presence of a mean wind shear, the dominant response at the intrinsic frequency $\hat{\omega}_{0}=k / \sqrt{k^{2}+l_{0}^{2}}$ will correspond to a broad range of frequencies due to Doppler shifting $\left(\omega=U k+\hat{\omega}_{0}\right)$, inducing the broad maximum of $\overline{u w}(c)$ (solid line) in Fig. 7. The effect of diffusion is minimized in this case, as the response spectrum consists of a broad range of wavelengths rather than a narrow band of slow short wavelength waves susceptible to diffusion or radiative damping. Consequently, the distribution of $\overline{u w}$ with $c$ has imprints of both critical level filtering and of other dynamical effects linked to the interaction of the distributed forcing with the mean flow that cannot be simply interpreted with arguments based on the Eliassen-Palm theorem, which is violated within the forcing region. Calculation of the outgoing wave action flux distribution with phase speeds $F_{a}(\omega)=\overline{u w}(\omega) / k$ with and without the shear layer (not presented) showed the same enhancement of upstream propagating waves. We note that, in this case of distributed forcing, extraction of more momentum is realized in the presence of shear even for large Richardson numbers, as opposed to the localized forcing considered in the previous section. The exhibited overall increase of the momentum flux carried by upstream propagating gravity waves in the presence of environmental wind shear was also noticed by previous studies (Beres et al. 2004; Alexander et al. 2004). In the Beres et al. (2004) study of convectively generated gravity waves above squall lines, the specified heating had a few dominant frequencies and they attributed most of the increase to the obstacle effect. They also noted that the contribution of nonzero frequencies could be larger in the presence of many more forcing frequencies, as was in fact verified in our calculation with broadband forcing.

Nevertheless, the largest dynamical effect of the shear is shown in the distribution of $\overline{p w}$ with phase speeds (lower right panel), where the maximum $\overline{p w}$ attained exceeds the corresponding one for a motionless atmosphere by a factor of 7 , leading to an integrated energy flux over all frequencies larger by a factor of 5.5.

\footnotetext{
${ }^{2}$ Note that, if the forcing had a half sine wave structure in the vertical, the largest forced wavenumber would be $l_{0}=\pi$ and the heating would therefore project most strongly onto a wave with vertical wavelength twice the depth of the heating as shown by Salby and Garcia (1987). This is not always true for monochromatic sources, where the horizontal extent of the source plays a significant role as found by Holton et al. (2002).
}
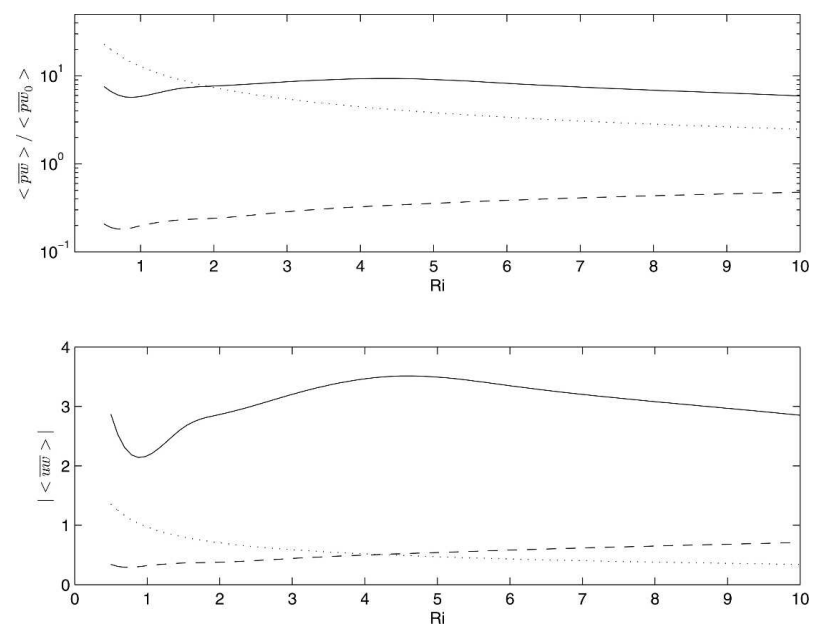

FIG. 8. Ensemble (upper) energy flux $\langle\overline{p w}\rangle$ and (lower) momentum flux $\langle\overline{u w}\rangle$ calculated at $z=1$ as a function of the Richardson number for $k=0.1$ (dotted line), $k=2$ (solid line) and $k=10$ (dashed line). The energy flux is scaled by the corresponding flux $\overline{p w}_{0}$ in the absence of the shear flow.

The phase speed, at which the maximum energy flux is attained, is $c=-0.16$. By substituting $l_{0}=20 \pi$ into the dispersion relation, we found that it corresponds to a wave generated at the lower end of the shear region, that is, the one that can exploit the whole depth of the shear region in order to grow transiently. As this wave propagates upward, its wavenumber decreases in the manner described in section 3 and it emerges from the upper part of the shear layer having a vertical wavelength $\lambda_{z}=2.2$, approximately twice the depth of the heating. This result was found to be weakly dependent on $\mathrm{Ri}, k$, and $l_{0}$ for large Richardson numbers. The implication is that, even though our source has fine scales, the structures that carry most of the energy eventually emerge from the shear layer having a scale comparable to that of the shear.

The dependence of the fluxes on the strength of the shear is illustrated in Fig. 8, where the ensemble momentum and energy fluxes at $z=1$ are plotted as a function of the Richardson number for three values of the horizontal wavenumber $[k=0.1$ (dotted line), $k=$ 2 (solid line), and $k=10$ (dashed line)] and is the result of two counteracting effects: The larger growth achieved for stronger shear and the reduction of the width of the region subject to interaction and propagation for lower Richardson numbers, ${ }^{3}$ because waves coming below $z=1 / 2-\sqrt{\mathrm{Ri}} / k$ are trapped. For small

\footnotetext{
${ }^{3}$ Following the same arguments presented in the previous section, waves can continue propagation in the upper region of uniform velocity only if they are excited in the region $1 / 2-\sqrt{\mathrm{Ri}} / k \leq$ $z \leq 1 / 2$.
} 
horizontal wavenumbers $(k=0.1)$, the effective region coincides with the whole shear layer regardless of the Richardson number and the fluxes increase monotonically as the shear becomes stronger. For large horizontal wavenumbers $(k=10)$, this effective region is less than half of the shear zone and its width is reduced as the Richardson number gets lower, leading to decreasing fluxes with decaying $\mathrm{Ri}$. In the intermediate case $(k=2),\langle\overline{p w}\rangle$ increases until it reaches a maximum at $\mathrm{Ri}=4.5$. For lower Richardson numbers, the larger energy growth achieved for stronger shear is inadequate to overcome the reduced leakage of wave activity and $\langle\overline{u w}\rangle$ and $\langle\overline{p w}\rangle$ drop for Ri $\langle 4.5$. Similar conclusions on the dependence of the width of the effective region on the horizontal wavenumber $k$ were reached by Lott (1997). It is also worth noting that in the inviscid limit the fluxes would grow without bound as $k \rightarrow 0$ since the response is inversely proportional to $k$. But even a small amount of diffusion (or Newtonian cooling) attenuates large horizontal wavelengths that have very small group velocity $\left[u_{g}=k l\left(k^{2}+l^{2}\right)^{3 / 2} \rightarrow 0\right.$ as $k$ $\rightarrow 0]$ and leads to maximum momentum and energy fluxes at $k_{\max }=0.75$ and $\mathrm{Ri}_{\text {max }}=0.5\left(\right.$ for $\left.\mathrm{Re}_{s}=10^{5}\right)$, as shown in Fig. 9 where the energy (upper panel) and momentum (lower panel) fluxes are plotted as a function of horizontal wavenumber $k$ and Richardson number. Finally, note that the thick black line in the upper panel of Fig. 9, which corresponds to a value of 1, shows that for $k>6$ the shear acts more like a weakly leaking "black box" of wave activity, rather than an efficient emitter.

\section{b. Optimal emission of gravity wave power from the shear region}

The largest energy fluxes that can be achieved by a single forcing function, are the ones forced by the stochastic optimals (Farrell and Ioannou 1996). The only difference here is that the stochastic optimals should be identified as the complete set of forcing functions of unit energy, resulting in the largest energy flux away from the shear layer at statistical equilibrium, rather than resulting in the largest equilibrium energy inside a certain region. To calculate them we first cast the equation governing the perturbation dynamics in terms of the new variable $\mathbf{y}=\mathbf{M}^{1 / 2} \mathbf{x}$, where $\mathbf{M}$ is the energy metric matrix, and the governing equations are then transformed to

$$
\frac{d \mathbf{y}}{d t}=\mathbf{A}_{\mathbf{m}} \mathbf{y}+\mathbf{F}_{\mathbf{m}} \mathbf{f}(t),
$$

where $\mathbf{A}_{\mathbf{m}}$ is given by

$$
A_{\mathbf{m}}=\mathbf{M}^{1 / 2} \mathbf{A M}^{-1 / 2}
$$
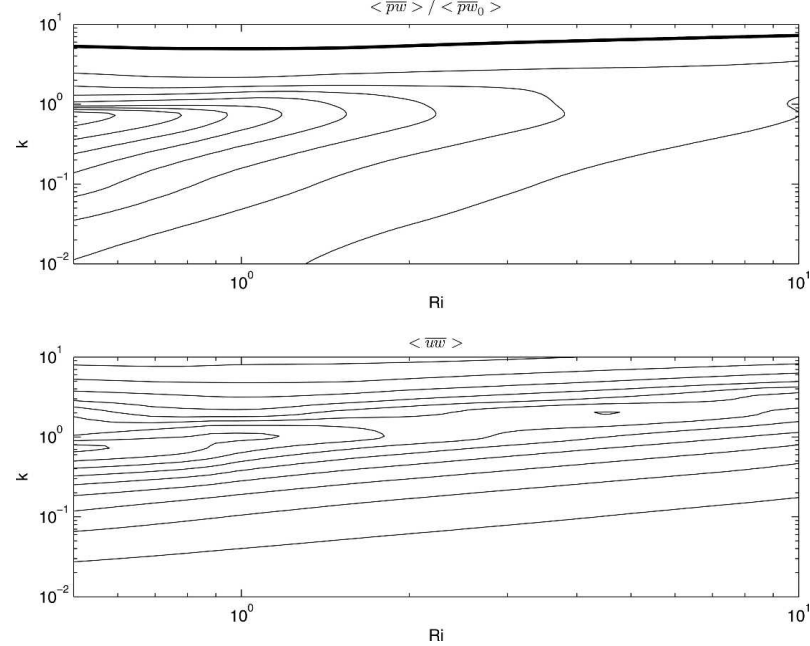

FIG. 9. (upper) Ensemble energy flux $\langle\overline{p w}\rangle$ calculated at $z=1$ and scaled by the corresponding flux $\left\langle\overline{p w}_{0}\right\rangle$ in the absence of the shear flow, as a function of the Richardson number Ri and the horizontal wavenumber $k$. The contour intervals are 5 with the innermost contour having the maximum value of 40 . The contour of 1 is also denoted by the black thick line. (lower) Integrated momentum flux $\langle\overline{u w}\rangle$ over all frequencies at $z=1$, as a function of $\mathrm{Ri}$ and $k$. All values shown are negative, the contour intervals are 0.5 (nondimensional units) with the innermost contour having the minimum value of -4.5 .

and $\mathbf{F}_{\mathbf{m}}$ is $\mathbf{F}_{\mathbf{m}}=\mathbf{M}^{1 / 2} \mathbf{F}$. The forced solution of (36) is

$$
\mathbf{y}(t)=\int_{0}^{t} e^{\mathbf{A}_{\mathbf{m}}(t-s)} \mathbf{F}_{\mathbf{m}} \mathbf{f}(s) d s .
$$

We are interested in the largest energy flux at $z=1$ (located outside the shear region), so to achieve that we introduce the projector matrix $\mathbf{M}_{\mathbf{T}}$, which has as its only nonzero element, the diagonal element that corresponds to the point $z=1$. We have already seen that the pressure $p$ and the vertical velocity $w$ can be expressed in terms of $\mathbf{x}$ using the linear operators $\mathbf{P}$ and W. Consequently the energy flux at $z=1$ at equilibrium is given by

$$
\begin{aligned}
\left\langle\overline{\mathbf{p w}^{\dagger}}\right\rangle & =\left\langle(\mathbf{W} \mathbf{x})^{\dagger} \mathbf{M}_{\mathbf{T}} \mathbf{P} \mathbf{x}\right\rangle=\left\langle\mathbf{y}^{\dagger}\left(\mathbf{M}^{-1 / 2}\right)^{\dagger} \mathbf{W}^{\dagger} \mathbf{M}_{\mathbf{T}} \mathbf{P M}^{-1 / 2} \mathbf{y}\right\rangle \\
& =\left\langle\mathbf{y}^{\dagger} \mathbf{P}_{\mathbf{w}} \mathbf{y}\right\rangle,
\end{aligned}
$$

where

$$
\mathbf{P}_{\mathbf{w}}=\left(\mathbf{M}^{-1 / 2}\right)^{\dagger} \mathbf{W}^{\dagger} \mathbf{M}_{\mathbf{T}} \mathbf{P} \mathbf{M}^{-1 / 2}
$$

and $\mathbf{y}$ is given by (38). Thus at equilibrium

$$
\left\langle\overline{\mathbf{p w}^{\dagger}}\right\rangle=\left\langle\mathbf{y}^{\dagger} \mathbf{P}_{\mathbf{w}} \mathbf{y}\right\rangle=\mathbf{F}_{\mathbf{m}}^{\dagger} \mathbf{B} \mathbf{F}_{\mathbf{m}},
$$

where

$$
\mathbf{B}=\int_{0}^{t} e^{\mathbf{A}_{\mathbf{m}}^{\dagger}(t-s)} \mathbf{P}_{\mathbf{w}} e^{\mathbf{A}_{\mathbf{m}}(t-s)} d s
$$



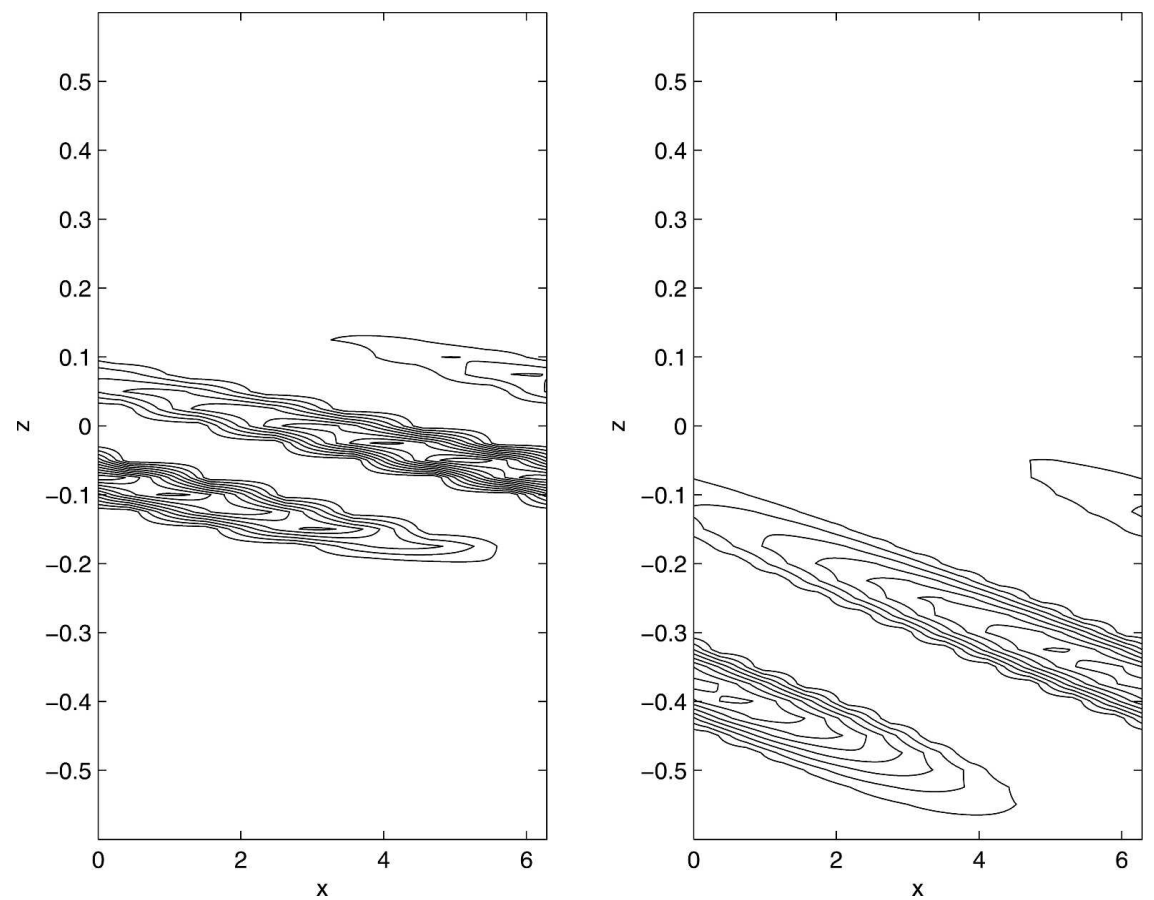

FIG. 10. (left) Structure of the first stochastic optimal (buoyancy forcing function) for $\mathrm{Ri}=$ 0.5 and $k=1$, which accounts for $24 \%$ of the resulting energy flux. (right) Structure of the first stochastic optimal (buoyancy forcing function) for $\mathrm{Ri}=10$ and $k=1$, which accounts for $26 \%$ of the resulting energy flux. The vorticity forcing functions are similar to the buoyancy forcing functions and are not shown.

By differentiating (42) we obtain

$$
\frac{d \mathbf{B}}{d t}=\mathbf{A}_{\mathbf{m}}^{\dagger} \mathbf{B}+\mathbf{B A}_{\mathbf{m}}+\mathbf{P}_{\mathbf{w}} .
$$

If $\mathbf{A}_{\mathbf{m}}$ is asymptotically stable, the system reaches statistical equilibrium and in the limit of $t \rightarrow \infty, d \mathbf{B} / d t \rightarrow 0$ and Eq. (43) becomes

$$
\mathbf{A}_{\mathbf{m}}^{\dagger} \mathbf{B}_{\infty}+\mathbf{B}_{\infty} \mathbf{A}_{\mathbf{m}}+\mathbf{P}_{\mathbf{w}}=0 .
$$

This Lyapunov equation determines the stochastic optimal matrix $\mathbf{B}_{\infty}$. From Eq. (41) it is seen that the orthogonal eigenvectors (the stochastic optimals) of the Hermitian $\mathbf{B}_{\infty}$ order according to their eigenvalue the power emitted from the shear region. Specifically the eigenvector of $\mathbf{B}_{\infty}$ with largest eigenvalue is the forcing structure that produces the largest gravity wave flux, which can be emitted from the shear region.

In Fig. 10 we plot the structure of the first stochastic optimal for $\mathrm{Ri}=0.5$ (left panel) and $\mathrm{Ri}=10$ (right panel). Both have the expected tilt against the shear that allows excitation of waves to favorably interact with the mean shear flow. For low Richardson numbers the effective region of excitation is toward the upper half of the shear region as propagation is suppressed. However, when $\mathrm{Ri}=10$ the optimal excitation is lo- cated toward the bottom of the shear region because in this case the perturbation can take advantage of the whole shear region for its growth. If, for each value of Richardson number $\mathrm{Ri}$ and zonal wavenumber $k$, we force with the first stochastic optimal and calculate the resulting ensemble energy flux $\langle\overline{p w}\rangle$ and then repeat the same calculation for an atmosphere at rest (forcing with the corresponding stochastic optimals), we can quantify the intensification of the gravity wave flux due to the shear. This ratio shown as a function of Ri and $k$ in Fig. 11. The transient growth mechanism accounts for up to 30 times larger fluxes compared to the uniform flow case, revealing that intermittently shear layers may be very powerful emitters of gravity waves.

\section{c. The case of temporally correlated stochastic forcing}

Although a temporally uncorrelated stochastic forcing allows the exploration of the response of a dynamical system over the full range of frequencies and wavenumbers without bias, in reality the spectrum of excited perturbations is broad but not white. The first step toward a more realistic description of the random forcing is to consider the effect of a finite temporal correlation 


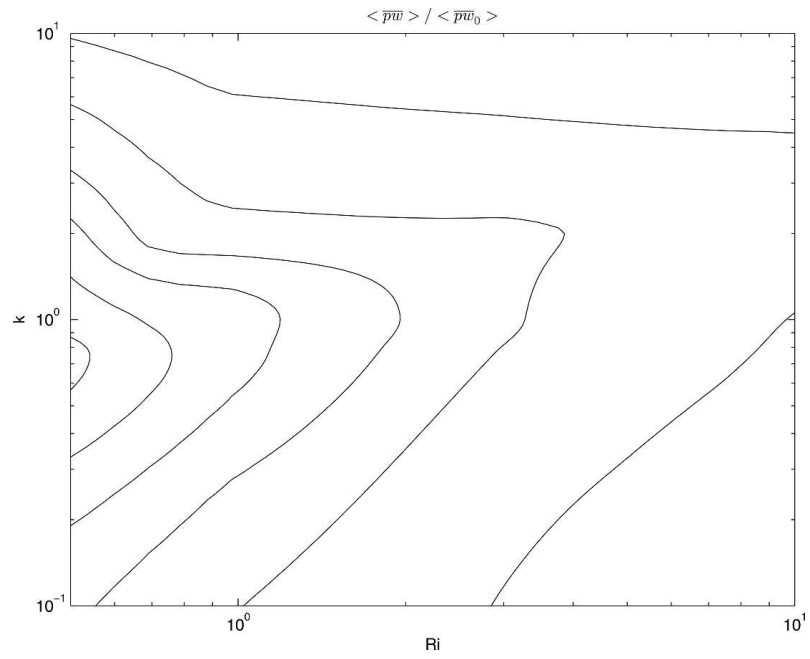

FIG. 11. Ensemble energy flux $\langle\overline{p w}\rangle$ obtained by the first stochastic optimal for every Richardson number and horizontal wavenumber, as a function of $\mathrm{Ri}$ and $k$. The flux is calculated at $z=1$ and scaled by the corresponding flux $\left\langle{\overline{p w_{0}}}_{0}\right\rangle$ obtained by the corresponding first stochastic optimal in the absence of the shear flow. The contour interval is 5 with the innermost contour having the maximum value of 30 .

in the forcing. For that purpose we consider forcing with exponentially decaying temporal correlations:

$$
\left\langle f\left(t_{1}\right) f\left(t_{2}\right)\right\rangle=\frac{\alpha}{2} e^{-\alpha\left(t_{2}-t_{1}\right)}
$$

with $\alpha=1 / \tau$, where $\tau$ is the autocorrelation time. Such a forcing is produced by the stochastic equation:

$$
\frac{d f}{d t}=-\alpha f+\alpha \xi(t),
$$

which is forced by a delta correlated white noise $\xi$ with zero mean and unit covariance:

$$
\langle\xi(t)\rangle=0, \quad\left\langle\xi\left(t_{1}\right) \xi\left(t_{2}\right)\right\rangle=\delta\left(t_{1}-t_{2}\right) .
$$

The total variance of each component of the correlated noise, $\left\langle f^{2}\right\rangle$, can be expressed in terms of its power spectrum, $F(\omega)$, as

$$
\left\langle f^{2}\right\rangle=\frac{1}{2 \pi} \int_{-\infty}^{\infty} F(\omega) d \omega,
$$

where

$$
F(\omega)=\frac{\alpha^{2}}{\alpha^{2}+\omega^{2}} .
$$

As $\alpha \rightarrow \infty$, the power spectrum $F(\omega)$ becomes the constant power spectrum of a delta correlated white noise in which all the frequencies are equally excited. For finite $\alpha$ the spectrum is red, decreasing at high frequen- cies as $\omega^{-2}$. This falloff of the red spectrum suppresses the excitation of high-frequency waves. For a given autocorrelation time $\tau$, the impact of the redness of the forcing on the spectrum of the excited gravity waves is found to strongly depend on the wavenumber of the waves forced.

In the left panel of Fig. 12, we plot the total integrated energy flux as a function of the autocorrelation time $\tau$ for waves with zonal wavenumber $k=0.1$ (dashed line) and $k=1$ (solid line). The outgoing flux for $k=1$ is strongly suppressed because the frequency at which the maximum response is attained (see upper right panel of Fig. 12, where the $\overline{p w}$ frequency spectra is plotted for $k=1$ ) lies outside the window of frequencies that are forced with significant power [refer to the dashed line which corresponds to the graph of $F(\omega)]$. On the other hand, the weak dependence of the energy flux for $k=0.1$ on the redness of the forcing, stems from the fact that the frequency at which the maximum response is attained (see lower right panel of Fig. 12, where the $\overline{p w}$ frequency spectra is plotted for $k=0.1$ ) is within the range of frequencies that are strongly forced [the dashed line corresponds again to the graph of $F(\omega)]$.

\section{Conclusions}

We have investigated the emission of gravity wave power from a stochastically forced shear layer. We first treated the propagation properties of waves excited by a monochromatic point forcing located in the middle of the shear region and then the properties of waves that result from a point source that is temporally varying as a white noise process. We have shown that low intrinsic frequency waves are always favorably excited. When the wavenumber $k$ of the waves is such that $1 / 2 \leq$ $\sqrt{\mathrm{Ri}} / k$, there is appreciable leakage of easterly momentum away from the shear layer accompanied with westward momentum deposition inside the shear region due to critical level filtering. This case would materialize for waves with horizontal wavelengths greater than $8 \mathrm{~km}$ for a typical wind shear of $V_{0}=30 \mathrm{~m} \mathrm{~s}^{-1}$ over $H=10 \mathrm{~km}$ and typical tropospheric static stability $N=0.012 \mathrm{~s}^{-1}$. Owing to the universal Orr mechanism that governs the wave-mean flow interactions and leads to transient energy growth, the outgoing waves have a wave energy flux 3 times larger than the emitted wave power in the absence of shear. Shorter waves $\left(\lambda_{x}<8\right.$ $\mathrm{km})$ that satisfy $\sqrt{\mathrm{Ri}} / k<1 / 2$ are trapped in the shear layer, in agreement with the findings of Lott (1997), and deposit their momentum at their critical level within the shear region.

The same qualitative results obtain when the shear 

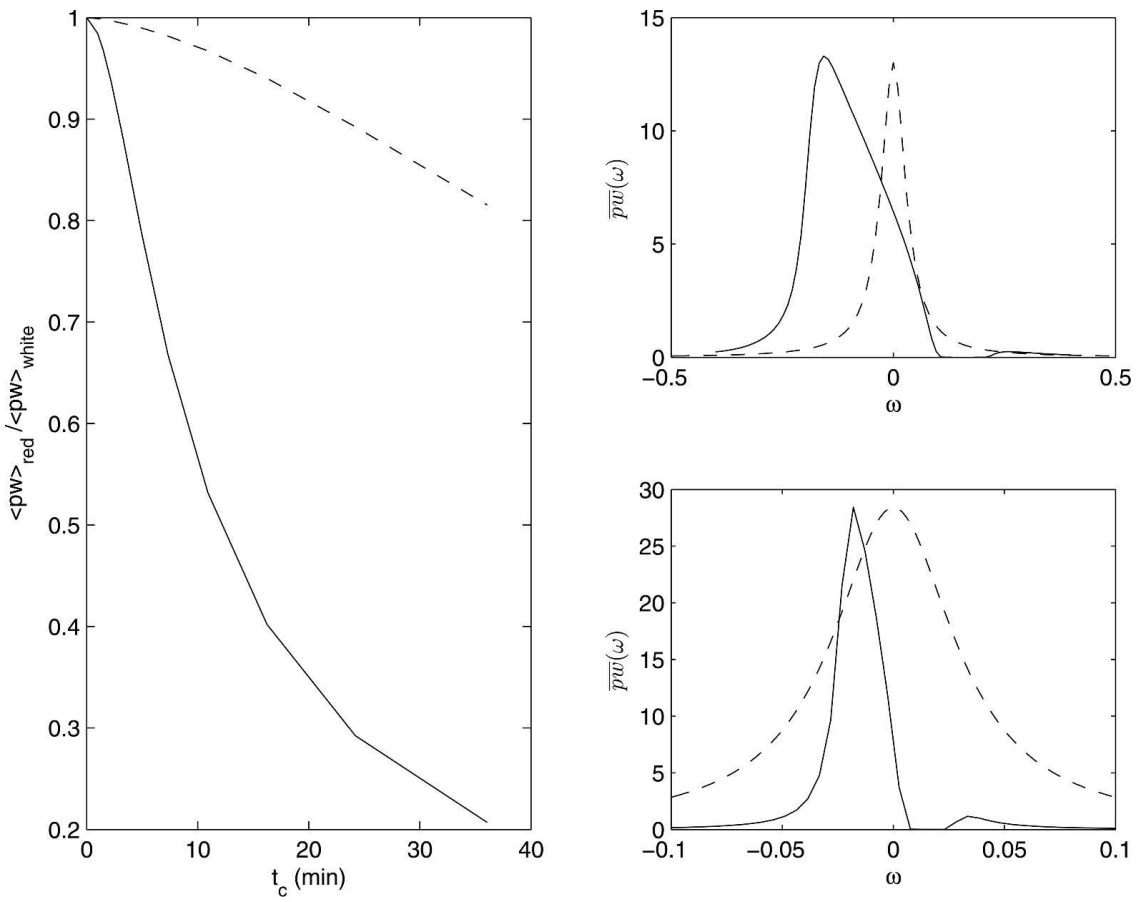

FIG. 12. (left) Effect of correlation time on the ensemble energy flux $\langle\overline{p w}\rangle_{\text {red }}$ for $k=1$ (solid line) and $k=0.1$ (dashed line). The flux is calculated at $z=1$ and scaled by the corresponding flux $\langle\overline{p w}\rangle_{\text {white }}$ for uncorrelated forcing. The Richardson number is $\mathrm{Ri}=10$. (right) Distribution of $\langle\overline{p w}\rangle$ at $z=1$ with frequencies for (upper right) $k=1$ and (lower right) $k=0.1$. The power spectrum $F(\omega)=\alpha^{2} /\left(\alpha^{2}+\omega^{2}\right)$, where $\alpha=1 /(30 \mathrm{~min})$, is also plotted (dashed line) for reference and is normalized to have at $\omega=0$ the maximum value of $\overline{p w}$ in each case.

layer is forced by a spatially extended thermal or vorticity stochastic forcing. However, the phase speed spectra of wave momentum and energy flux are modified in this case due to wave interference and Doppler shifting effects. Even though such an unbiased forcing equally drives all perturbations, it strongly couples with emitted waves leaning against the shear and carrying momentum upstream. While for large Richardson numbers momentum is carried away from the forced region by waves having a wide range of vertical wavenumbers, most of the energy is carried by the ones having vertical wavelengths in the range $1.5 H<\lambda_{z}<3 H$ (for a shear region of depth of $H \mathrm{~km}$ ), regardless of their zonal wavenumber. It was shown that, although initially shorter waves are favored by such sources, the combination of wave refraction and transient amplification as they shear over during propagation leads to waves finally emerging from the upper part of the shear layer that have scales comparable to the shear layer depth.

We also find that for $k<6$ (corresponding to horizontal wavelengths $\lambda_{x}>H \mathrm{~km}$ ) the shear region is a highly efficient emitter, even for large Richardson numbers, as the excited perturbations exploit the potential for nonnormal transient growth. Moreover, increasing shear, that is, decreasing Richardson number, leads to enhanced wave activity radiating away that reaches a global maximum at $k=0.75\left(\lambda_{x}=8.4 \mathrm{Hkm}\right)$. A proper measure for the efficiency of the shear layer as an emitter is the maximum outgoing wave energy flux that can be emitted from normalized excitations. This occurs when the forcing assumes the structure of the appropriate stochastic optimal. We calculated that, at best, the optimal energy flux that can be emitted from a shear region is 30 times greater than the flux emitted when the waves are excited by the corresponding stochastic optimal in the absence of shear. Such a large amplification factor might not always occur but shows the true potential of shear for enhancing gravity wave activity, even in the absence of instabilities.

The emission of wave energy is curtailed when the forcing does not excite waves with phase lines tilted against the shear or when the waves cannot escape from the shear layer. It can also be curtailed when the stochastic forcing becomes adequately correlated in time. In that case the degree of reduction of wave power depends on the horizontal wavenumber of the excited waves. Red noise forcing was found to strongly influence waves with horizontal wavenumber larger than 
$k=0.5\left(\lambda_{x}=12.5 H \mathrm{~km}\right)$ while lower wavenumbers are unaffected, even for correlation times $O(1 \mathrm{~h})$.

This study offers additional insight on the dynamical effect of shear regions in the emission of gravity wave power. The shear flow does not only passively filter and refract the emitted wave spectrum, but also actively participates in the gravity wave emission in conjunction with the distributed forcing. This interaction leads to enhanced radiated momentum fluxes but more importantly to enhanced gravity wave energy fluxes with the aid of transient growth exhibited by the excited perturbations during propagation within the shear region.

Acknowledgments. The authors thank three anonymous reviewers for their useful comments and numerous suggestions, which helped to improve the manuscript. It is also a pleasure to acknowledge helpful discussions with Prof. Brian Farrell. NB's research was supported by NSF ATM-0432463.

\section{APPENDIX A}

\section{Propagation of Wave Packets in Stratified Shear Flow}

Suppose that we launch a wave packet of the form

$$
\left[\psi_{0}(\xi, \eta), \rho_{0}(\xi, \eta)\right]=[1,0] f(\eta) e^{i k_{0} \xi+i l_{0} \eta}
$$

where $f=\exp \left\{-\left[\left(\eta-\eta_{0}\right) / \delta \eta\right]^{2}\right\}$ and $\delta \eta$ is the width of packet. The general solution to Eqs. (8)-(9) with initial conditions

$$
[\psi(\xi, \eta, \tau=0), \rho(\xi, \eta, \tau=0)]=\left[\psi_{0}(\xi, \eta), \rho_{0}(\xi, \eta)\right]
$$

can be written as

$$
\psi(\xi, \eta, \tau)=\frac{1}{\sqrt{2 \pi}} \int_{-\infty}^{\infty} \hat{\psi}(l, \tau) e^{i k_{0} \xi+i l \eta} d l
$$

with $\hat{\psi}(l, 0)=\hat{\psi}_{0}(l)$, where $\hat{\psi}_{0}(l)$ satisfies the initial condition

$$
\hat{\psi}_{0}(l)=\frac{1}{\sqrt{2 \pi}} \int_{-\infty}^{\infty} f(\eta) e^{-i\left(l-l_{0}\right) \eta} d \eta=\hat{f}\left(l-l_{0}\right) .
$$

The evolution of $\hat{\psi}(l, \tau)$ can be determined from the perturbation vorticity equation (11), which accepts for large Richardson numbers the following WKB solution:

$$
\hat{\zeta}(l, \tau)=A \frac{1}{\sqrt{\omega(\tau)}} e^{i \int_{0}^{\tau} \omega(s) d s}+B \frac{1}{\sqrt{\omega(\tau)}} e^{-i \int_{0}^{\tau} \omega(s) d s},
$$

where $A=\left(k_{0} / \sqrt{4 \omega(0)}\right)\left(k_{0} \hat{\psi}_{0}(l) / \omega(0)-\hat{\rho}_{0}(l)\right), B=$ $\left(k_{0} / \sqrt{4 \omega(0)}\right)\left(k_{0} \hat{\psi}_{0}(l) / \omega(0)+\hat{\rho}_{0}(l)\right)$ are amplitudes related to the initial conditions [the Fourier component of the initial density perturbation $\hat{\rho}_{0}(l)$ is defined similarly with $\left.\hat{\psi}_{0}(l)\right]$ and $\omega=k_{0} / \sqrt{k_{0}^{2}+\left(l-k_{0} \tau / \sqrt{\mathrm{Ri}}\right)^{2}}$ is the time dependent frequency of the wave. Therefore, having assumed that initially there is no density perturbation, $\hat{\rho}_{0}(l)=0$, we obtain

$$
\begin{aligned}
\hat{\psi}(l, t)= & \frac{\hat{\zeta}(l, \tau)}{k_{0}^{2}+\left(l-k_{0} \tau / \sqrt{\mathrm{Ri}}\right)^{2}} \\
= & \frac{1}{2} \frac{\left(k_{0}^{2}+l^{2}\right)^{3 / 4}}{\left(k_{0}^{2}+\left(l-k_{0} \tau / \sqrt{\mathrm{Ri}}\right)^{2}\right)^{3 / 4}} \hat{\psi}_{0}(l) \\
& \times\left(e^{i \int_{0}^{\tau} \omega(s) d s}+e^{-i \int_{0}^{\tau} \omega(s) d s}\right) \\
= & a(l, \tau) \hat{\psi}_{0}(l)\left(e^{i b(l, \tau)}+e^{-i b(l, \tau)}\right),
\end{aligned}
$$

where $a(l, \tau)=(1 / 2)\left(k_{0}^{2}+l^{2}\right)^{3 / 4}\left[k_{0}^{2}+\left(l-k_{0} \tau / \sqrt{\mathrm{Ri}}\right)^{2}\right]^{-3 / 4}$ and $b(l, \tau)=\int_{0}^{\tau} \omega(s) d s$. So the first term of Eq. (A5) becomes

$$
\begin{aligned}
\psi(\xi, \eta, \tau) & =\frac{1}{\sqrt{2 \pi}} \int_{-\infty}^{\infty} a(l, \tau) \hat{\psi}_{0}(l) e^{i b(l, \tau)} e^{i k_{0} \xi+i l \eta} d l \\
& =\frac{1}{\sqrt{2 \pi}} \int_{-\infty}^{\infty} a(l, \tau) \hat{f}\left(l-l_{0}\right) e^{i b(l, \tau)} e^{i k_{0} \xi+i l \eta} d l .
\end{aligned}
$$

Expanding all functions near $l_{0}$, yields

$$
\begin{aligned}
\psi(\xi, \eta, \tau)= & \frac{1}{\sqrt{2 \pi}} \int_{-\infty}^{\infty}\left(\hat{f}(0)+\left.\partial_{l} \hat{f}\right|_{0}\left(l-l_{0}\right)+\cdots\right) \\
& \times\left(\left.a\right|_{l_{0}}+\left.\partial_{l} a\right|_{l_{0}}\left(l-l_{0}\right)+\cdots\right) E d l, \quad
\end{aligned}
$$

where

$$
\begin{aligned}
E= & \exp \left[i k_{0} \xi+i l_{0} \eta+i\left(l-l_{0}\right) \eta+\left.i b\right|_{l_{0}}+\left.i \partial_{l} b\right|_{l_{0}}\left(l-l_{0}\right)\right. \\
& \left.+\left.i \partial_{l}^{2} b\right|_{l_{0}}\left(l-l_{0}\right)^{2} / 2+\cdots\right] .
\end{aligned}
$$

Changing variables, $\tilde{l}=l-l_{0}$, Eq. (A6) becomes 


$$
\begin{aligned}
& \psi(\xi, \eta, \tau)=\frac{e^{i k_{0} \xi+i l_{0} \eta+i b\left(l_{0}, \tau\right)}}{\sqrt{2 \pi}} \int_{-\infty}^{\infty}\left(\left.\hat{f}(0) a\right|_{l_{0}}+\left.\hat{f}(0) \partial_{l} a\right|_{l_{0}} \tilde{l}+O\left(\tilde{l}^{2}\right)\right) \times e^{\left.i \partial \vec{\partial} b\right|_{l_{0}} \tilde{l}+i \partial_{l}^{2} b||_{l_{0}} \tilde{z}^{2} / 2^{+}+\cdots e^{i \eta_{\eta}}} d \tilde{l} \\
& =\frac{\hat{f}(0) e^{i k_{0} \xi+i l_{0} \eta+i b\left(l_{0}, \tau\right)}}{\sqrt{2 \pi}}\left(a\left(l_{0}, \tau\right) \int_{-\infty}^{\infty} e^{i \partial\left|b l_{l} \tilde{l}+i \partial_{l}^{2} b\right|_{l_{0}} \tilde{l}^{2} / 2 e^{i \eta_{l}}} d \tilde{l}+\left.\partial_{l} a\right|_{l_{0}} \int_{-\infty}^{\infty} \tilde{l}^{i \partial l_{l} b l_{0} \tilde{l}+i \partial_{l}^{2} b l_{l_{0}} \tilde{l}^{2} / 2 e^{i \eta_{l}}} d \tilde{l}\right)+O\left(\tilde{l}^{2}\right) .
\end{aligned}
$$

If we choose $\eta=-\left.\partial_{l} b\right|_{l_{0}}$, then we get

$$
\begin{aligned}
\psi(\xi, \eta, \tau)= & e^{i k_{0} \xi+i l_{0} \eta+i b\left(l_{0}, \tau\right)} \hat{\psi}_{0}\left(l_{0}\right) a\left(l_{0}, \tau\right) e^{i \pi / 4} \sqrt{1 /\left.\partial_{l}^{2} b\right|_{l_{0}}} \\
& +O\left[\left(l-l_{0}\right)^{2}\right] .
\end{aligned}
$$

So the center of the wave packet is moving according to

$$
\begin{aligned}
\hat{z}(\tau)= & \hat{z}(0)+\sqrt{\operatorname{Ri}}\left(\frac{1}{\sqrt{k_{0}^{2}+\left(l_{0}-k_{0} \tau / \sqrt{\mathrm{Ri}}\right)^{2}}}\right. \\
& \left.-\frac{1}{\sqrt{k_{0}^{2}+l_{0}^{2}}}\right),
\end{aligned}
$$

and the group velocity will be given by

$$
c_{g_{z}}=\frac{k_{0}\left(l_{0}-k_{0} \tau / \sqrt{\mathrm{Ri}}\right)}{\left(k_{0}^{2}+\left(l_{0}-k_{0} \tau / \sqrt{\mathrm{Ri}}\right)^{2}\right)^{3 / 2}} .
$$

Following the same analysis for the second term, we get

$$
\begin{aligned}
\hat{z}(\tau)= & \hat{z}(0)-\sqrt{\operatorname{Ri}}\left(\frac{1}{\sqrt{k_{0}^{2}+\left(l_{0}-k_{0} \tau / \sqrt{\mathrm{Ri}}\right)^{2}}}\right. \\
& \left.-\frac{1}{\sqrt{k_{0}^{2}+l_{0}^{2}}}\right)
\end{aligned}
$$

and the group velocity will be given by

$$
c_{g_{z}}=-\frac{k_{0}\left(l_{0}-k_{0} \tau / \sqrt{\mathrm{Ri}}\right)}{\left[k_{0}^{2}+\left(l_{0}-k_{0} \tau / \sqrt{\mathrm{Ri}}\right)^{2}\right]^{3 / 2}} .
$$

\section{APPENDIX B}

\section{Response to Point Thermal Forcing in a Stratified Shear Layer}

We consider the inviscid Eqs. (5)-(6) forced by the point thermal forcing:

$$
f_{t}(x, z, t)=\delta(z) e^{i k x} e^{-i k c t}
$$

We obtain the Green's function of the inviscid Eqs. (5)-(6) by assuming solutions of the form

$$
[\psi(x, z, t), \rho(x, z, t)]=[\hat{\psi}(z), \hat{\rho}(z)] e^{i k x} e^{-i k c t} .
$$

The inviscid version of Eqs. (5)-(6) then become after elimination of $\hat{\rho}$ :

$$
\begin{gathered}
\frac{d^{2} \hat{\psi}}{d z^{2}}+\left(\frac{\mathrm{Ri}}{(U(z)-c \sqrt{\mathrm{Ri}})^{2}}-\frac{d^{2} U}{d z^{2}} \frac{1}{(U(z)-c \sqrt{\mathrm{Ri}})}-k^{2}\right) \hat{\psi} \\
=\frac{\mathrm{Ri}}{i k(U(z)-c \sqrt{\mathrm{Ri}})^{2}} \delta(z),
\end{gathered}
$$

where $U(z)$ is given by (19). The solution of (B3) is

$$
\hat{\psi}(z)=\left\{\begin{array}{cl}
F e^{i l_{u}\left(z-z_{0}\right)}, & \text { for } \quad z \geq z_{0} \\
D \sqrt{k(z-c \sqrt{\mathrm{Ri}})} I_{\mu}\left[k(z-c \sqrt{\mathrm{Ri})}]+E \sqrt{k(z-c \sqrt{\mathrm{Ri}})} I_{-\mu}[k(z-c \sqrt{\mathrm{Ri}})],\right. & \text { for } \quad 0 \leq z<z_{0} \\
B \sqrt{k(z-c \sqrt{\mathrm{Ri}})} I_{\mu}\left[k(z-c \sqrt{\mathrm{Ri})}]+C \sqrt{k(z-c \sqrt{\mathrm{Ri}})} I_{-\mu}[k(z-c \sqrt{\mathrm{Ri}})],\right. & \text { for } \quad-z_{0} \leq z<0 \\
A e^{i l_{l}\left(z+z_{0}\right)}, & \text { for } z<-z_{0}
\end{array}\right.
$$

where $l_{l}$ and $l_{u}$ are the square roots of $\mathrm{Ri} /\left(1 / 2+\right.$ having $\Re\left(l_{l}\right)\left(c \sqrt{\mathrm{Ri}}+z_{0}\right)>0, \mathfrak{I}\left(l_{l}\right)<0, \Re\left(l_{u}\right)(c \sqrt{\mathrm{Ri}}-$ $c \sqrt{\mathrm{Ri}})^{2}-k^{2}$ and $\mathrm{Ri} /(1 / 2-c \sqrt{\mathrm{Ri}})^{2}-k^{2}$, respectively, $\left.z_{0}\right)<0, \mathfrak{I}\left(l_{u}\right)>0$ (as boundedness or radiation condi- 
tions must be satisfied at $z \rightarrow \mp \infty$, respectively), $\mu=$ $\sqrt{1 / 4-\mathrm{Ri}}$ and $I_{ \pm \mu}$ is the modified Bessel function of the second kind of order $\mu$.

Continuity of displacement and pressure at $z=$ $\pm z_{0}$ implies continuity of $\hat{\psi} /(U(z)-c \sqrt{\mathrm{Ri}})$ and $(U(z)-c \sqrt{\mathrm{Ri}}) d \hat{\psi} / d z-(d U / d z) \hat{\psi}$ at $z= \pm z_{0}$, respectively. Integration of (B3) twice from $z=0^{-}$to $z=0^{+}$to obtain two interface conditions at $z=0$ yields the following algebraic equation for the amplitudes

$$
\left[\mathbf{B}_{1}, \mathbf{B}_{2}\right] \mathbf{Y}=\mathbf{B}_{3},
$$

where $\mathbf{Y}=[A, B, C, D, E, F]^{\mathrm{T}}$ and

$$
\begin{aligned}
& \mathbf{B}_{1}=\left(\begin{array}{ccc}
1 & -a_{+} & -a_{-} \\
i l l_{l}\left(z_{0}+c \sqrt{\mathrm{Ri}}\right) & -\left(z_{0}+c \sqrt{\mathrm{Ri}}\right) k \delta a_{+}-a_{+} & -\left(z_{0}+c \sqrt{\mathrm{Ri}}\right) k \delta a_{-}-a_{-} \\
0 & -c_{+} & -c_{-} \\
0 & -k \delta c_{+} & -k \delta c_{-} \\
0 & 0 & 0 \\
0 & 0 & 0
\end{array}\right) \text {, } \\
& \mathbf{B}_{2}=\left(\begin{array}{ccc}
0 & 0 & 0 \\
0 & 0 & 0 \\
d_{+} & d_{-} & 0 \\
k \delta d_{+} & k \delta d_{-} & 0 \\
-b_{+} & -b_{-} & 1 \\
-\left(z_{0}-c \sqrt{\mathrm{Ri}}\right) k \delta b_{+}+b_{+} & -\left(z_{0}-c \sqrt{\mathrm{Ri}}\right) k \delta b_{-}+b_{-} i l_{u}\left(z_{0}-c \sqrt{\mathrm{Ri}}\right)
\end{array}\right) \text {, }
\end{aligned}
$$

and

$$
\mathbf{B}_{\mathbf{3}}=\left[0,0,0,1 /\left(i k c^{2}\right), 0,0\right]^{\mathrm{T}} \text {. }
$$

The coefficients in the expressions above are, when $-z_{0}<c \sqrt{\mathrm{Ri}}<0$,

$$
\begin{aligned}
& a_{ \pm}=-i e^{\mp i \mu \pi} \sqrt{z_{l}} I_{ \pm \mu}\left(z_{l}\right), \quad b_{ \pm}=\sqrt{z_{u}} I_{ \pm \mu}\left(z_{u}\right), \\
& c_{ \pm}=\sqrt{\mid k c \sqrt{\mathrm{Ri}} I_{ \pm \mu}}(|k c \sqrt{\mathrm{Ri}}|), \\
& d_{ \pm}=c_{ \pm}, \quad \delta a_{ \pm}=i e^{\mp i \mu \pi}\left(\frac{I_{ \pm \mu}\left(z_{l}\right)}{2 \sqrt{z_{l}}}+\sqrt{z_{l}}\left(\left.\frac{d I_{ \pm \mu}}{d z}\right|_{z_{l}}\right),\right. \\
& \delta b_{ \pm}=\frac{I_{ \pm \mu}\left(z_{u}\right)}{2 \sqrt{z_{u}}}+\left.\sqrt{z_{u}} \frac{d I_{ \pm \mu}}{d z}\right|_{z_{u}}, \\
& \delta c_{ \pm}=\frac{I_{ \pm \mu}(|k c \sqrt{\mathrm{Ri}}|)}{2 \sqrt{|k c \sqrt{\mathrm{Ri}}|}}+\left.\sqrt{|k c \sqrt{\mathrm{Ri}}|} \frac{d I_{ \pm \mu}}{d z}\right|_{|k c \sqrt{\mathrm{Ri}}|},
\end{aligned}
$$$$
\delta d_{ \pm}=\delta c_{ \pm} z_{l}=k\left(z_{0}+c \sqrt{\mathrm{Ri}}\right), z_{u}=k\left(z_{0}-c \sqrt{\mathrm{Ri}}\right) .
$$

When $0<c \sqrt{\mathrm{Ri}}<z_{0}$ the coefficients are

$$
a_{ \pm}=\sqrt{z_{l}} I_{ \pm \mu}\left(z_{l}\right), \quad b_{ \pm}=\sqrt{z_{u}} I_{ \pm \mu}\left(z_{u}\right),
$$$$
c_{ \pm}=\sqrt{k c \sqrt{\mathrm{Ri}}} I_{ \pm \mu}(k c \sqrt{\mathrm{Ri}}),
$$$$
d_{ \pm}=-i e^{\mp i \mu \pi} \sqrt{k c \sqrt{\mathrm{Ri}}} I_{ \pm \mu}(k c \sqrt{\mathrm{Ri}})
$$$$
\delta a_{ \pm}=-\left(\frac{I_{ \pm \mu}\left(z_{l}\right)}{2 \sqrt{z_{l}}}+\left.\sqrt{z_{l}} \frac{d I_{ \pm \mu}}{d z}\right|_{z_{l}}\right)
$$$$
\delta b_{ \pm}=\frac{I_{ \pm \mu}\left(z_{u}\right)}{2 \sqrt{z_{u}}}+\left.\sqrt{z_{u}} \frac{d I_{ \pm \mu}}{d z}\right|_{z_{u}},
$$$$
\delta c_{ \pm}=-\left(\frac{I_{ \pm \mu}(k c \sqrt{\mathrm{Ri}})}{2 \sqrt{k c \sqrt{\mathrm{Ri}}}}+\left.\sqrt{k c \sqrt{\mathrm{Ri}}} \frac{d I_{ \pm \mu}}{d z}\right|_{k c \sqrt{\mathrm{Ri}}}\right),
$$

$$
\begin{aligned}
\delta d_{ \pm}= & i e^{\mp i \mu \pi}\left(\frac{I_{ \pm \mu}(k c \sqrt{\mathrm{Ri}})}{2 \sqrt{k c \sqrt{\mathrm{Ri}}}}\right. \\
& \left.+\left.\sqrt{k c \sqrt{\mathrm{Ri}}} \frac{d I_{ \pm \mu}}{d z}\right|_{k c \sqrt{\mathrm{Ri}}}\right)
\end{aligned}
$$

$$
z_{l}=k\left(z_{0}+c \sqrt{\mathrm{Ri}}\right), \quad z_{u}=k\left(z_{0}-c \sqrt{\mathrm{Ri}}\right) .
$$


Otherwise, they are

$$
\begin{aligned}
a_{ \pm}= & \sqrt{z_{l}} I_{ \pm \mu}\left(z_{l}\right), \quad b_{ \pm}=\sqrt{z_{u}} I_{ \pm \mu}\left(z_{u}\right), \\
c_{ \pm}= & \sqrt{|k c \sqrt{\mathrm{Ri}}|} I_{ \pm \mu}(|k c \sqrt{\mathrm{Ri}}|), \quad d_{ \pm}=c_{ \pm}, \\
\delta a_{ \pm}= & -\operatorname{sgn}(c)\left(\frac{I_{ \pm \mu}\left(z_{l}\right)}{2 \sqrt{z_{l}}}+\left.\sqrt{z_{l}} \frac{d I_{ \pm \mu}}{d z}\right|_{z_{l}}\right), \\
\delta b_{ \pm}= & -\operatorname{sgn}(c)\left(\frac{I_{ \pm \mu}\left(z_{u}\right)}{2 \sqrt{z_{u}}}+\sqrt{z_{u}}\left|\frac{d I_{ \pm \mu} \mid}{d z}\right|_{z_{u}}\right), \\
\delta c_{ \pm}= & -\operatorname{sgn}(c)\left(\frac{I_{ \pm \mu}(|k c \sqrt{\mathrm{Ri}}|)}{2 \sqrt{|k c \sqrt{\mathrm{Ri}}|}}\right. \\
& \left.+\left.\sqrt{|k c \sqrt{\mathrm{Ri}}|} \frac{d I_{ \pm \mu} \mid}{d z}\right|_{k c \sqrt{\mathrm{Ri} \mid}}\right) \\
\delta d_{ \pm}= & \delta c_{ \pm}, \quad z_{l}=k\left|z_{0}+c \sqrt{\mathrm{Ri}}\right|, \\
z_{u}= & k\left|z_{0}-c \sqrt{\mathrm{Ri}}\right| .
\end{aligned}
$$

The resulting momentum and energy fluxes are, respectively,

$$
\begin{aligned}
\overline{u w}(c) & =\frac{1}{2} \Re\left(u w^{*}\right)=\frac{1}{2} \Re\left(i k \partial_{z} \hat{\psi} \hat{\psi}^{*}\right), \\
\overline{p w}(c) & =\frac{1}{2} \Re\left(p w^{*}\right) \\
& =\frac{1}{2} \Re\left[-(U / \sqrt{\mathrm{Ri}}-c) \hat{u} \hat{w}^{*}+\frac{i}{k \sqrt{\mathrm{Ri}}} \frac{d U}{d z} \hat{w} \hat{w}^{*}\right] \\
& =\frac{1}{2} \Re\left[-i k(U / \sqrt{\mathrm{Ri}}-c) \partial_{z} \hat{\psi} \hat{\psi}^{*}\right]
\end{aligned}
$$

Following the same steps for a motionless atmosphere, the solution of (B3) is

$$
\hat{\psi}(z)=\left\{\begin{array}{cl}
A_{0} e^{-\operatorname{sgn}(c) i l z}, & \text { for } z \geq 0 \\
B_{0} e^{\operatorname{sgn}(c) i l z}, & \text { for } z<0
\end{array},\right.
$$

where $A_{0}=B_{0}=1 /\left(2 k l c^{2} \operatorname{sgn}(c)\right)$ are obtained by the continuity conditions at $z=0$. The corresponding momentum and energy fluxes are

$$
\begin{aligned}
\overline{u w}_{0}(c) & =\frac{1}{2} \Re\left(i k \frac{\partial \hat{\psi}}{\partial z} \hat{\psi}^{*}\right) \\
& =\left\{\begin{array}{cl}
\operatorname{sgn}(c)\left(k^{2}+l^{2}\right)^{2} /(8 k l), & \text { for } z \geq 0 \\
-\operatorname{sgn}(c)\left(k^{2}+l^{2}\right)^{2} /(8 k l), & \text { for } \quad z<0
\end{array}\right.
\end{aligned}
$$

and

$$
\begin{aligned}
{\overline{p w_{0}}}_{(c)} & =\frac{1}{2} \Re\left(-i k(U / \sqrt{\mathrm{Ri}}-c) \frac{\partial \hat{\psi}}{\partial z} \hat{\psi}^{*}\right) \\
& =\left\{\begin{array}{cc}
|c|\left(k^{2}+l^{2}\right)^{2} /(8 k l), & \text { for } \quad z \geq 0 \\
-|c|\left(k^{2}+l^{2}\right)^{2} /(8 k l), & \text { for } \quad z<0
\end{array}\right.
\end{aligned}
$$

respectively.

\section{REFERENCES}

Achatz, U., and G. Schmitz, 2006a: Shear and static instability of inertia-gravity wave packets: Short-term modal and nonmodal growth. J. Atmos. Sci., 63, 397-413.

$\longrightarrow$, and — 2006b: Optimal growth in inertia-gravity wave packets: Energetics, long-term development, and threedimensional structure. J. Atmos. Sci., 63, 414-434.

Alexander, M. J., P. T. May, and J. H. Berres, 2004: Gravity waves generated by convection in the Darwin area during the Darwin Area Wave Experiment. J. Geophys. Res., 109, D20S04, doi:10.1029/2004JD004729.

Beres, J. H., M. J. Alexander, and J. R. Holton, 2002: Effects of tropospheric wind shear on the spectrum of convectively generated gravity waves. J. Atmos. Sci., 59, 1805-1824.

,$- \ldots$, and $—, 2004$ : A method of specifying the gravity wave spectrum above convection based on latent heating properties and background wind. J. Atmos. Sci., 61, 324-337.

Chimonas, G., and J. R. Grant, 1984: Shear excitation of gravity waves. Part II: Upscale scattering from Kelvin-Helmholtz waves. J. Atmos. Sci., 41, 2278-2288.

Clark, T. L., T. Hauf, and J. P. Kuettner, 1986: Convectively forced internal gravity waves: Results from two-dimensional numerical experiments. Quart. J. Roy. Meteor. Soc., 112, 899925.

Eliassen, A., and E. Palm, 1961: On the transfer of energy in stationary mountain waves. Geofys. Publ., 22, 1-23.

Farrell, B. F., 1988: Optimal excitation of neutral Rossby waves. $J$. Atmos. Sci., 45, 163-172.

_ 1989: Optimal excitation of baroclinic waves. J. Atmos. Sci., 46, 1193-1206.

_ waves. J. Atmos. Sci., 50, 4044-4057.

— stratified shear flow. J. Atmos. Sci., 50, 2201-2214.

_ , and _ 1996: Generalized stability theory. Part I: Autonomous operators. J. Atmos. Sci., 53, 2025-2040.

Fritts, D. C., 1984: Shear excitation of atmospheric gravity waves. Part II: Nonlinear radiation from a free shear layer. J. Atmos. Sci., 41, 524-537.

_ fects in the middle atmosphere. Rev. Geophys., 41, 1003, doi:10.1029/2001RG000106.

Hartman, R. J., 1975: Wave propagation in a stratified shear flow. J. Fluid Mech., 71, 89-104.

Holton, J. R., J. H. Beres, and X. Zhou, 2002: On the vertical scale 
of gravity waves excited by localized thermal forcing. $J$. Atmos. Sci., 59, 2019-2023.

Lindzen, R. S., 1966: On the relation of wave behavior to source strength and distribution in a propagating medium. J. Atmos. Sci., 23, 630-632.

— in stably stratified shear flow. J. Fluid Mech., 151, 189-217.

Lott, F., 1997: The transient emission of propagating gravity waves by a stably stratified shear layer. Quart. J. Roy. Meteor. Soc., 123, 1603-1619.

Salby, M. L., and R. R. Garcia, 1987: Transient response to localized episodic heating in the Tropics. Part I: Excitation and short-time near-field behavior. J. Atmos. Sci., 44, 458-498.
Scinocca, J. F., and R. Ford, 2000: The nonlinear forcing of largescale internal gravity waves by stratified shear instability. $J$. Atmos. Sci., 57, 653-672.

Sutherland, B. R., 1996: Dynamic excitation of internal gravity waves in the equatorial oceans. J. Phys. Oceanogr., 26, 23982419.

, 2000: Internal wave reflection in uniform shear. Quart. J. Roy. Meteor. Soc., 126, 3255-3286.

— the middle atmosphere from a model tropospheric jet. J. Atmos. Sci., 52, 3214-3235.

, and P. F. Linden, 1998: Internal wave excitation from stratified flow over a thin barrier. J. Fluid Mech., 377, 223-252. 